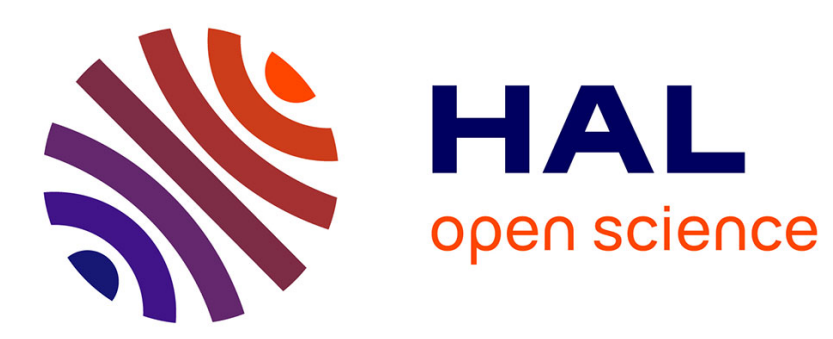

\title{
A modal strategy devoted to the hidden state variables method with large interfaces
}

Pierre Ropars, Christophe Desceliers

\section{To cite this version:}

Pierre Ropars, Christophe Desceliers. A modal strategy devoted to the hidden state variables method with large interfaces. Computational Mechanics, 2015, 55 (5), pp.805-818. 10.1007/s00466-015-1148z . hal-01140558

\section{HAL Id: hal-01140558 \\ https://hal.science/hal-01140558}

Submitted on 8 Apr 2015

HAL is a multi-disciplinary open access archive for the deposit and dissemination of scientific research documents, whether they are published or not. The documents may come from teaching and research institutions in France or abroad, or from public or private research centers.
L'archive ouverte pluridisciplinaire HAL, est destinée au dépôt et à la diffusion de documents scientifiques de niveau recherche, publiés ou non, émanant des établissements d'enseignement et de recherche français ou étrangers, des laboratoires publics ou privés. 


\title{
A modal strategy devoted to the Hidden State Variables Method with large interfaces
}

\author{
Pierre Ropars · Christophe Desceliers
}

Received: date / Accepted: date

\begin{abstract}
In many mechanical engineering applications, the interactions of a structure through its boundary is modelled by a dynamic boundary stiffness matrix. Nevertheless, it is well known that the solution of such computational model is very sensitive to the modelling uncertainties on the dynamic boundary stiffness matrix. In a recent work, the "hidden state variables method" is used to identify mass, stiffness and damping matrices associated with a given deterministic dynamic boundary stiffness matrix which can be constructed by using experimental measurements. Such an identification allows the construction of the probabilistic model of a random boundary stiffness matrix by substituting those identified mass, stiffness and damping matrices by random matrices. Nevertheless, the numerical cost of the "hidden state variables method" increases drastically with the dimension (number of degrees of freedom) of the interface. We then propose an enhanced approach which consists in a truncated spectral representation of the displacements on the boundary and with a partition of the frequency band of analysis. A collection of mass, stiffness and damping matrices is then identified for each sub-frequency band of analysis. A probabilistic model is constructed in substituting each of those matrices by random matrices. A numerical application is proposed.
\end{abstract}

P. Ropars

MSME Université Paris-Est Marne-la-Vallée; 5 Bd Descartes, 77454 Marne-la-Vallée cedex 2, France

E-mail: pierre.ropars@gmail.com

C. Desceliers

MSME Université Paris-Est Marne-la-Vallée; 5 Bd Descartes, 77454 Marne-la-Vallée cedex 2, France

E-mail: christophe.desceliers@univ-paris-est.fr
Keywords Finite elements method · Hidden state variables method · Random matrices - Random dynamic boundary stiffness matrix

\section{Introduction}

The uncertainties quantification of complex mechanical systems is a challenge for engineers and researchers. This paper deals with an inverse method in the Fourier space for the uncertainty quantification in a structural linear visco-elasto dynamics system in low frequency band.

The mechanical system under study is composed by $\Omega_{2}$ which is coupled with $\Omega_{1}$. It is assumed that a numerical model of $\Omega_{2}$ can be constructed, while it is not the case for $\Omega_{1}$. The coupling between $\Omega_{1}$ and $\Omega_{2}$ is performed on an interface $\Gamma$. For example, it is the case for the design of a building $\left(\Omega_{2}\right)$ whose foundations are coupled with an unknown soil $\left(\Omega_{1}\right)$. Nevertheless, it is usual to model the dynamical response of $\Omega_{1}$ on the interface $\Gamma$ by a boundary dynamical stiffness matrix which can be obtained by experimental measurements or by numerical computations.

The numerical derivation of the boundary stiffness matrix requires the resolution of a classical mixed boundary value problem. A large set of usual methods is therefore usable, among which the finite-elements method [1] is the most often used. When the domain $\Omega_{1}$ becomes large, or unbounded, an artificial boundary has to be introduced. The truncation of the domain means that waves would reflect on the artificial boundary and pollute the solution in the domain $\Omega_{2}$. Many techniques have been derived in the literature to prevent this problem. Some of them are reviewed in [2,3], or in special journal issues on absorbing boundary conditions [4-6]. 
These techniques can be gathered into three groups: global absorbing boundary conditions, local absorbing boundary conditions, and absorbing layer. Besides these finite-elements-based methods stands the boundary elements method [7]. Then, it appears quite natural for the computation of the boundary stiffness matrix, since the formulation is directly performed on the boundary and in case of an unbounded domain, the radiation conditions are directly taken into account. The scaled boundary elements method [8] is an alternative approach which does not require a fundamental solution, and only requires the meshing of the boundary and enforces naturally the radiation condition. The main requirement for this method is a particular type of radial symmetry of the domain. When the boundary stiffness matrices of $\Omega_{1}$ and $\Omega_{2}$ are computed separately, the mobility method allows the response of both $\Omega_{1}$ and $\Omega_{2}$ to be simulated when the two domains are coupled [9-11]. In an other point of view, the subsystem $\Omega_{1}$ transposes on $\Gamma$ a distant boundary condition. Several authors introduce a Dirichlet-to-Neumann map to describe, in the time domain, the external forces due to $\Omega_{1}$ that are applied to $\Gamma[2,12]$.

In addition, many methods have been proposed to reduce the number of degrees of freedom in a model [23]. We can cite the condensation techniques [24], or the truncated expansion techniques which are very popular. They are proposed in any computational problem which is concerned by large numerical cost. In the scope of soil-structure interaction problem, the boundary finite element method has received several significant advances $[25,26]$. Recent developments in the bridging scale method proposed an efficient response to a dynamical interface problem [27]. Also, in the time domain and in the scope of the Dirichlet-to-Neumann mapping, several authors have developed efficient methods [28-30]. This paper is concerned by a problem in the Fourier domain, and the method used hereinafter is based on an ad-hoc truncated expansion of eigenfunctions.

However, in the framework of the robust conception of $\Omega_{2}$, it is necessary to quantify the uncertainties induced by the lack of knowledge on $\Omega_{1}$. In this aim, a probabilistic approach is very efficient, and a probabilistic model of the uncertainties related to $\Omega_{1}$ can be constructed by modelling the boundary dynamical stiffness matrix as a random matrix. In the last decade, a new probabilistic approach has been developed in linear visco-elasto dynamic in order to model the uncertainties related to the modelling of a mechanical system in constructing an ad hoc probabilistic model of the mass, damping and stiffness matrices [13,14]. In [15-17], such a probabilistic approach has been applied to construct the model of the random boundary dynamical stiffness matrix. It has been achieved with the identification of mass, damping and stiffness matrices corresponding to a dynamical stiffness matrix for which the Shur complement is equal to the boundary dynamical stiffness matrix of $\Omega_{1}$.

The hidden state variables method $[15,16,18,19]$ is an efficient method devoted to such an identification. Among all the degrees of freedom associated with the identified mass, damping and stiffness matrices, those that do not belong to interface $\Gamma$ are called hidden variables. It should be noted that they are not physical degrees of freedom, but "meta" degrees of freedom which give an equivalent description of the dynamical response of $\Omega_{1}$ on the interface. It is assumed that (1) the unknown complete dynamical model of $\Omega_{1}$ is a second-order differential equation; (2) the boundary dynamic stiffness matrix is symmetric and continuous on the frequency band of analysis $B_{\mathrm{f}}$; (3) the mass, damping and stiffness matrices to be identified are real, symmetric and positive-definite. These assumptions have physical interpretation, and assure the preservation of principles of reciprocity [20] and causality [21].

Despite numerous advantages, some numerical difficulties occur with an extensive use of the hidden state variables method: the stability and the numerical cost. In [22] and [19], the stability issue has been addressed. The numerical cost increases with the dimension of the problem (number of degrees of freedom of the interface). Indeed, computational time increases drastically when the dimension is greater than 10, even with a reasonable frequency band of analysis. The extension of the hidden state variables method in high dimension remains a challenge. This would allow a realistic mechanical system with a high dimension to be studied. Consequently, we propose hereinafter a formulation that allows such an extension without modification of the numerical algorithms used in the hidden state variable method in order to carry out the identification. This is achieved by introducing a reduced representation of the displacements field on interface $\Gamma$ for sub-frequency bands of analysis included in $B_{\mathrm{f}}$.

In the second section of the paper, the boundary dynamic stiffness matrix and the dynamic equation of $\Omega_{2}$ are presented. The following section deals with the reduction of the problem and its decomposition on several sub-frequency bands of analysis. Then, in the fourth section, the hidden state variables method is briefly presented. Then, a probabilistic model for the random boundary dynamic stiffness matrix is formulated for each sub-frequency bands of analysis. It should be noted that the proposed approach yields a set statistically independent random mass, damping and stiffness matrices for each sub-frequency bands of analysis. Some nu- 


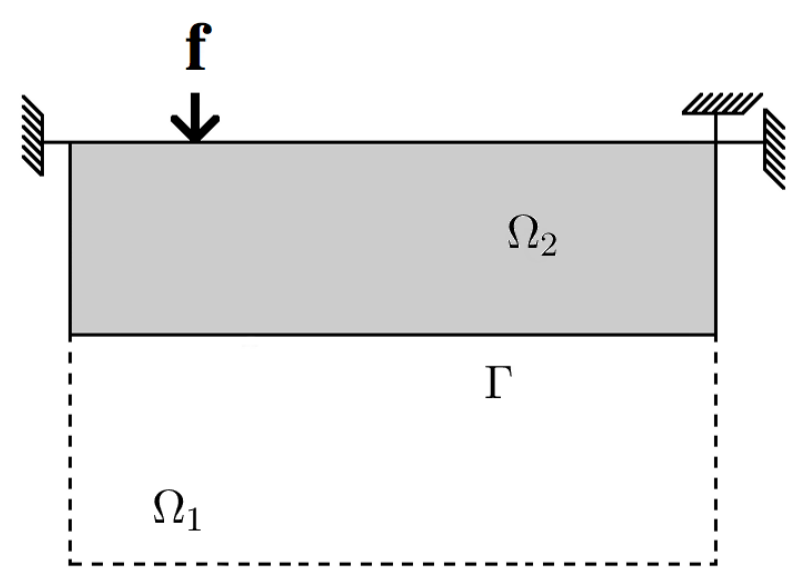

Fig. 1 Geometry of domain $\Omega$.

merical applications are given in section 5.3, 5.4, 5.5, 5.6 and 6.2 in order to study the efficiency of the proposed approach.

\section{Boundary dynamic stiffness matrix and dynamic equation of $\Omega_{2}$}

We consider a structure modelled in Fourier space by a visco-elastic medium and that is occupying a bounded domain denoted by $\Omega$. Domain $\Omega$ is made up of two distinct sub-domains denoted by $\Omega_{1}$ and $\Omega_{2}$. Let $\Gamma$ be the interface between $\Omega_{1}$ and $\Omega_{2}$ (see Fig. 1). A finite-element interpolation basis is used to calculate a numerical approximation of the displacement field $\mathbf{x} \mapsto \mathbf{u}(\mathbf{x}, \omega)$ in $\Omega_{2}$ in which $\mathbf{x}$ is the vector of coordinates of any generic point in $\Omega_{2}$ and $\omega$ is the angular frequency. For all $\omega$ in the frequency band of analysis $B_{\mathrm{f}}$, let $\mathbf{u}_{2}(\omega) \in \mathbb{R}^{n_{2}}$ be the vector of all the degrees of freedom of the finite-element interpolation and let the mass, damping and stiffness real $\left(n_{2} \times n_{2}\right)$ matrices be denoted by $\left[M_{2}\right],\left[D_{2}\right]$ and $\left[K_{2}\right]$. For all frequency $\omega$ in $B_{\mathrm{f}}$, the dynamic stiffness matrix $\left[A_{2}(\omega)\right]$ of domain $\Omega_{2}$ is then written as

$$
\left[A_{2}(\omega)\right]=-\omega^{2}\left[M_{2}\right]+\jmath \omega\left[D_{2}\right]+\left[K_{2}\right] .
$$

The coupling between the two visco-elastic media occupying $\Omega_{1}$ and $\Omega_{2}$ is taken into account in constructing the boundary dynamic stiffness $\left[A_{\Gamma}(\omega)\right]$ associated with the displacements on interface $\Gamma$ and which is a symmetric $\left(n_{\Gamma} \times n_{\Gamma}\right)$ matrix where $n_{\Gamma}$ is the number of degree of freedom on $\Gamma$ for the finite element model of $\Omega_{2}$. Let us assume that the numbering of the degrees of freedom is such that $\mathbf{u}_{2}(\omega)$ is written as

$\mathbf{u}_{2}(\omega)=\left(\begin{array}{c}\mathbf{u}_{\Omega_{2}}(\omega) \\ \mathbf{u}_{\Gamma}(\omega)\end{array}\right)$ where $\mathbf{u}_{\Omega_{2}}(\omega)$ is the vector of the $n_{2}-n_{\Gamma}$ degrees of freedom related to nodes of the finite-element mesh in $\Omega_{2}$ and $\mathbf{u}_{\Gamma}(\omega)$ is the vector of the $n_{\Gamma}$ degrees of freedom related to the nodes on interface $\Gamma$. We then have, for all $\omega$ in $B_{\mathrm{f}}$,

$\left(\left[A_{2}(\omega)\right]+[A(\omega)]\right) \mathbf{u}_{2}(\omega)=\mathbf{f}(\omega)$,

where $\mathbf{f}(\omega) \in \mathbb{R}^{n_{2}}$ is the finite-element vector of the external forces applied on structure and in which the block diagonal matrix $[A(\omega)]$ is written as

$[A(\omega)]=\left(\begin{array}{ll}{\left[0_{2}\right]} & \\ & {\left[A_{\Gamma}(\omega)\right]}\end{array}\right)$,

where the $\left(n_{2}-n_{\Gamma} \times n_{2}-n_{\Gamma}\right)$ real matrix $\left[0_{2}\right]$ is the null matrix.

\section{Reduced representation of the displacement at the interface}

3.1 Spectral functional basis adapted for domain $\Omega_{2}$ with a coupled interface $\Gamma$

The main idea of the proposed approach is to reduce the number of degrees of freedom representing the displacements on interface $\Gamma$. Such a reduction is carried out in rewriting the dynamical system in using an $a d$ $h o c$ functional basis. A very efficient functional basis that is widely used in the literature consists in the eigenfunctions associated with the generalized eigenvalue problem for the whole domain $\Omega$. In our situation, the numerical model of sub-domain $\Omega_{1}$ is assumed to be not given. Another functional basis can be made up of the eigenfunctions associated with the generalized eigenvalue problem of sub-domain $\Omega_{2}$ with fixed interface $\Gamma$ or with free interface $\Gamma$. Nevertheless, the additional mass, damping and stiffness due to the coupling with sub-domain $\Omega_{1}$ are not taking into account. Consequently, a high number of eigenfunction should be used in order to represent accurately the displacement field on the interface. It is the reason why we introduce the following generalized eigenvalue problem: find eigenvectors $\phi_{\alpha} \in \mathbb{R}^{n_{2}}$ and eigenvalues $\lambda_{\alpha}$ such as

$\left[\mathbb{K}_{2}\right] \phi_{\alpha}=\lambda_{\alpha}\left[\mathbb{M}_{2}\right] \phi_{\alpha}$,

in which

$\left[\mathbb{K}_{2}\right]=\left[K_{2}\right]+\mathcal{R}\{[A(0)]\}$,

and

$\left[\mathbf{M}_{2}\right]=\left[M_{2}\right]+\frac{1}{c} \int_{B_{\mathrm{f}}} \mathcal{R}\{[A(\omega)]-[A(0)]\} d \omega$, 
where $\mathcal{R}\{[a]\}$ means the real part of any complex matrix $[a]$ and $c=-\int_{B_{\mathrm{f}}} \omega^{2} d \omega$. The additional terms in the right side of Eqs (6) and (7) corresponds to additional mass and stiffness due to the coupling of interface $\Gamma$ with sub-domain $\Omega_{1}$. Similarly, we introduce a damping matrix $\mathbb{D}_{2}$ defined as

$$
\left[\mathbb{D}_{2}(\omega)\right]=\left[D_{2}\right]+\mathcal{I}\{[A(\omega)]\},
$$

where $\mathcal{I}\{[a]\}$ means the imaginary part of any complex matrix $[a]$. The additional term in the right side of Eq (8) corresponds to an additional damping due the coupling of interface $\Gamma$ with subdomain $\Omega_{1}$. It is assumed that matrices $\left[\mathrm{M}_{2}\right]$ and $\left[\mathrm{K}_{2}\right]$ are definite-positive. Consequently, this generalized eigenvalue problem yields an increasing sequence of eigenvalues $0<\lambda_{1} \leq \lambda_{2} \leq$ $\ldots \leq \lambda_{n_{2}}$, associated with the eigenvectors $\phi^{1}, \ldots$, $\phi^{n_{2}}$ in $\mathbb{R}^{n_{2}}$. For any given $\alpha=1, \ldots, n_{2}$, the eigenfrequency $\omega_{\alpha}($ in $\mathrm{rad} / \mathrm{s})$ is defined as $\omega_{\alpha}=\lambda_{\alpha}^{1 / 2}$. The numbering of the degrees of freedom is such that, for any $\alpha=1, \ldots, n_{2}$ eigenvector $\phi^{\alpha}$ is written as

$\phi^{\alpha}=\left(\begin{array}{c}\phi_{\Omega_{2}}^{\alpha} \\ \phi_{\Gamma}^{\alpha}\end{array}\right)$

where $\phi_{\Omega_{2}}^{\alpha}$ is the vector of the $n_{2}-n_{\Gamma}$ degrees of freedom related to nodes in $\Omega_{2}$ and $\phi_{\Gamma}^{\alpha}$ is the vector of the $n_{\Gamma}$ degrees of freedom related to the nodes on interface $\Gamma$. The set of vectors $\phi_{\Gamma}^{1}, \ldots, \phi_{\Gamma}^{n_{2}}$ can be used in order to construct a spectral representation of vector $\mathbf{u}_{\Gamma}$ but it should be noted that this set of vectors are linearly dependent and consequently, they do not define an ad hoc functional basis.

\subsection{Decomposition of the frequency band of analysis}

\section{$B_{\mathrm{f}}$}

For using the hidden state variables method with vectors $\phi_{\Gamma}^{1}, \ldots, \phi_{\Gamma}^{n_{2}}$ as spectral functional basis of the displacements on interface $\Gamma$, we introduce a decomposition of the frequency band of analysis $B_{\mathrm{f}}$ into $n_{\mathrm{f}}$ frequency bands $B_{1}, \ldots, B_{n_{\mathrm{f}}}$ which are not necessarily disjoints and such that $B_{\mathrm{f}}=\cup_{k=1}^{n_{\mathrm{f}}} B_{k}$. Let the $m_{k}$ eigenfrequencies $\omega_{k, 1} \leq \ldots \leq \omega_{k, m_{k}}$ be all the eigenfrequencies belonging to $B_{k}$. Then, for $k=1, \ldots, n_{\mathrm{f}}$, the construction of the $k$-th frequency band $B_{k}$ is such that

$\left[\omega_{k, 1}-\frac{H_{k, 1}}{2}, \omega_{k, m_{k}}+\frac{H_{k, m_{k}}}{2}\right] \subset B_{k}$,

where $H_{\alpha}=\pi \xi_{\alpha} \omega_{\alpha} \sqrt{1-\xi_{\alpha}^{2}}$ with $\xi_{\alpha}=D_{\alpha} / 2 \sqrt{M_{\alpha} K_{\alpha}}$ and $M_{\alpha}=\left(\phi^{\alpha}\right)^{T}\left[M_{2}\right] \phi^{\alpha}, D_{\alpha}=\left(\phi^{\alpha}\right)^{T}\left[\mathbb{D}_{2}\left(\omega_{\alpha}\right)\right] \phi^{\alpha}$ and $K_{\alpha}=\left(\phi^{\alpha}\right)^{T}\left[\mathbb{K}_{2}\right] \phi^{\alpha}$. Two special cases can be considered. The first special case is obtained with $n_{\mathrm{f}}=1$ $\left(k=1\right.$ and $\left.m_{k}=n_{2}\right)$ and the second special case is obtained with $m_{k}=1\left(n_{\mathrm{f}}=n_{2}\right.$ and $m_{k}=1$ for all $\left.1 \leq k \leq n_{2}\right)$. Beside these two cases, any other combination is also possible. Note that, when $m_{k}$ is constant for all $B_{k}$, the number of frequency bands $n_{f}$ is fixed. This configuration is used hereinafter in Sections 5 and 6.2. In order to ensure that all frequencies of $B_{\mathrm{f}}$ are contained at least in one $B_{k}$, each frequency band $B_{k}$ can be taken as :

$$
\begin{gathered}
B_{k}=\left[\left(\omega_{k-1, m_{k-1}}+\frac{H_{k-1, m_{k-1}}}{2}+\omega_{k, 1}-\frac{H_{k, 1}}{2}\right) / 2,\right. \\
\left.\left(\omega_{k, m_{k}}+\frac{H_{k, m_{k}}}{2}+\omega_{k+1,1}-\frac{H_{k+1,1}}{2}\right) / 2\right] .
\end{gathered}
$$

3.3 Reduced interface displacement model in each frequency band $B_{k}$

We introduce the $\left(n_{\Gamma} \times m_{k}\right)$ interface modal matrix $\left[\phi_{\Gamma, B_{k}}\right]$ which is such that its $\ell$-th column is vector $\phi_{\Gamma}^{k, \ell}$ (see Eq. 9) associated with $\omega_{k, \ell}$ which is the $\ell$-th eigenfrequency in $B_{k}$. An orthogonal basis is deduced by the singular value decomposition of matrix $\left[\phi_{\Gamma, B_{k}}\right]$ written as

$\left[\phi_{\Gamma, B_{k}}\right]=\left[U_{\Gamma, B_{k}}\right]\left[\Sigma_{\Gamma, B_{k}}\right]\left[V_{\Gamma, B_{k}}\right]^{T}$,

where $\left[U_{\Gamma, B_{k}}\right]$ is an unitary $\left(n_{\Gamma} \times n_{\Gamma}\right)$ matrix, $\left[\Sigma_{\Gamma, B_{k}}\right]$ is a $\left(n_{\Gamma} \times m_{k}\right)$ matrix whose only elements on its diagonal are non zero and where $\left[V_{\Gamma, B_{k}}\right]$ is an unitary $\left(m_{k} \times\right.$ $m_{k}$ ) matrix. It is assumed that the singular values of $\left[\phi_{\Gamma, B_{k}}\right]$ are listed in descending order on the diagonal of $\left[\Sigma_{\Gamma, B_{k}}\right]$. Consequently, we have the following block decomposition of $\left[U_{\Gamma, B_{k}}\right]$,

$\left[U_{\Gamma, B_{k}}\right]=\left(\left[U_{\Gamma, B_{k}}^{+}\right]\left[U_{\Gamma, B_{k}}^{0}\right]\right)$,

where the columns of $\left[U_{\Gamma, B_{k}}^{+}\right]$are all the vectors associated with all the non-zero singular values of $\left[\phi_{\Gamma, B_{k}}\right]$. Let $m_{B_{k}}^{+}$be the number of non-zero singular values of $\left[\phi_{\Gamma, B_{k}}\right]$. Note that, in general, $m_{B_{k}}^{+}=\min \left(n_{\Gamma}, m_{k}\right)$, but in the following $m_{B_{k}}^{+}=m_{k}$. For any given frequency $\omega$ in $B_{k}$, we introduce the $m_{B_{k}}^{+}$-order approximation $\mathbf{u}_{\Gamma, B_{k}}(\omega)$ of vector $\mathbf{u}_{\Gamma}(\omega)$ defined as

$\mathbf{u}_{\Gamma, B_{k}}(\omega)=\left[U_{\Gamma, B_{k}}^{+}\right] \mathbf{q}_{B_{k}}(\omega)$,

where $\mathbf{q}_{B_{k}}(\omega)$ is the vector of the $m_{B_{k}}^{+}$generalized interface displacements on interface $\Gamma$ for the frequency band $B_{k}$. For any given $\omega$ in $B_{k}$, we then have the following approximation $\mathbf{u}_{2, B_{k}}(\omega)$ of vector $\mathbf{u}_{2}(\omega)$,

$\mathbf{u}_{2, B_{k}}(\omega)=\left[P_{B_{k}}\right] \mathbb{u}_{2, B_{k}}(\omega)$,

in which the block diagonal matrix $\left[P_{B_{k}}\right]$ and the reduced representation $\mathbb{u}_{2, B_{k}}(\omega)$ of $\mathbf{u}_{2, B_{k}}$ are written as

$\left[P_{B_{k}}\right]=\left(\begin{array}{ll}{\left[I_{2}\right]} & \\ & {\left[U_{\Gamma, B_{k}}^{+}\right]}\end{array}\right) \quad$ and $\quad \mathbb{u}_{2, B_{k}}(\omega)=\left(\begin{array}{l}\mathbf{u}_{\Omega_{2}}(\omega) \\ \boldsymbol{q}_{B_{k}}(\omega)\end{array}\right)$ 
where $\left[I_{2}\right]$ is the $\left(n_{2}-n_{\Gamma} \times n_{2}-n_{\Gamma}\right)$ identity matrix. Multiplying each side of Eq. (3) by $\left[P_{B_{k}}\right]^{T}$ and in using Eq. (16), we then obtain

$$
\left(\left[A_{2, B_{k}}(\omega)\right]+\left[A_{B_{k}}(\omega)\right]\right) \mathbb{u}_{2, B_{k}}(\omega)=\mathbb{E}_{B_{k}}(\omega),
$$

in which $\mathbb{E}_{B_{k}}(\omega)=\left[P_{B_{k}}\right]^{T} \mathbf{f}(\omega)$ and where the square matrices of dimension $\left(n_{2}-n_{\Gamma}+m_{B_{k}}^{+}\right)\left[A_{2, B_{k}}(\omega)\right]$ and $\left[A_{B_{k}}(\omega)\right]$ are defined as

$$
\left[A_{2, B_{k}}(\omega)\right]=\left[P_{B_{k}}\right]^{T}\left[A_{2}(\omega)\right]\left[P_{B_{k}}\right],
$$

and

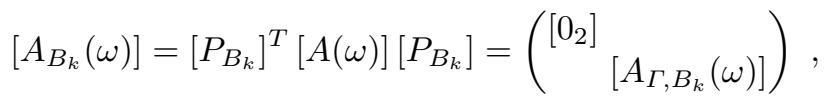

in which the symmetric $\left(m_{B_{k}}^{+} \times m_{B_{k}}^{+}\right)$matrix $\left[A_{\Gamma, B_{k}}(\omega)\right]=$ $\left[U_{\Gamma, B_{k}}^{+}\right]^{T}\left[A_{\Gamma}(\omega)\right]\left[U_{\Gamma, B_{k}}^{+}\right]$is the reduced boundary dynamic boundary stiffness matrix associated with the displacements on interface $\Gamma$.

\section{The hidden state variable method}

Hereinafter, a short overview of the hidden state variables method is presented. For more details, the reader can refer to $[15,16]$.

4.1 Algebraic representation of the boundary stiffness matrix $\left[A_{\Gamma}(\omega)\right]$

For a large range of engineering applications, boundary stiffness matrix $\left[A_{\Gamma}(\omega)\right]$ (see Eqs. (3) and (4)) is obtained in using experimental measurements in dynamics. It is the case when a numerical model of $\Omega_{1}$ is tricky to be constructed. The hidden states variables method can be used in order to identify a symmetric positive definite $\left(n_{1} \times n_{1}\right)$ matrix $\left[M_{1}\right]$ and two symmetric positive $\left(n_{1} \times n_{1}\right)$ matrices $\left[D_{1}\right]$ and $\left[K_{1}\right]$ such that

$$
\left[A_{\Gamma}(\omega)\right]=\left[A_{1 \Gamma}(\omega)\right]-\left[A_{1 c}(\omega)\right]\left[A_{1 h}(\omega)\right]^{-1}\left[A_{1 c}(\omega)\right]^{T},
$$

in which the symmetric $\left(n_{\Gamma} \times n_{\Gamma}\right)$ matrix $\left[A_{1 \Gamma}(\omega)\right]$, the $\left(n_{1}-n_{\Gamma} \times n_{\Gamma}\right)$ matrix $\left[A_{1 c}(\omega)\right]$ and the symmetric $\left(n_{1}-n_{\Gamma} \times n_{1}-n_{\Gamma}\right)$ matrix $\left[A_{1 h}(\omega)\right]$ are obtained from the block decomposition of the dynamic stiffness matrix $\left[A_{1}(\omega)\right]=-\omega^{2}\left[M_{1}\right]+\jmath \omega\left[D_{1}\right]+\left[K_{1}\right]$ written as

$$
\left[A_{1}(\omega)\right]=\left(\begin{array}{c}
{\left[A_{1 h}(\omega)\right]} \\
{\left[A_{1 c}(\omega)\right]^{T}\left[A_{1 c}(\omega)\right]} \\
{\left[A_{1 \Gamma}(\omega)\right]}
\end{array}\right)
$$

where the numbering of the degree of freedom are such that the $n_{\Gamma}$ last rows and the $n_{\Gamma}$ last columns of $\left[A_{1}(\omega)\right]$ are related to the $n_{\Gamma}$ degrees of freedom of nodes on $\Gamma$.

4.2 Step 1 : Interpolation of the given boundary dynamic stiffness matrix

The first step of the method consists in constructing an interpolation of $\omega \mapsto\left[A_{\Gamma}(\omega)\right]$. Boundary dynamic stiffness matrix is rewritten as $\left[A_{\Gamma}(\omega)\right]=\left[N^{\text {opt }}(\omega)\right] / q^{\text {opt }}(\omega)$ in which $\left[N^{\text {opt }}\right]$ belongs to $\mathcal{S}\left(n_{\Gamma}, d_{N}\right)$ and $q^{\text {opt }}$ belongs to $\mathcal{S}\left(1, d_{q}\right)$ where $\mathcal{S}(n, d)$ means the set of all polynomial functions of degree $d$ with values in the set of all the symmetric $(n \times n)$ matrices. The two polynomial functions $\left[N^{\text {opt }}\right]$ and $q^{\text {opt }}$ are then defined as the optimal functions solving an optimization problem. We then have, in [15],

$$
\left(\left[N^{\mathrm{opt}}\right], q^{\mathrm{opt}}\right)=\arg \min _{\substack{q \in \mathcal{S}\left(1, d_{q}\right) \\[N] \in \mathcal{S}\left(n_{\Gamma}, d_{N}\right)}} \varepsilon([N], q),
$$

with $d_{N}=d_{q}+2$ and where

$\varepsilon([N], q)=\int_{B_{\mathrm{f}}} c(\omega)^{2}\left\|\frac{[N(\omega)]}{q(\omega)}-\left[A_{\Gamma}(\omega)\right]\right\|_{\mathrm{F}}^{2} d \omega$,

and $\omega \mapsto c(\omega)$ is a regularizing function [31] and $\|\cdot\|_{F}$ is the Frobenius norm of symmetric matrices. The algorithm presented in [15] to solve Eq. (22) requires to perform the numerical orthogonalization of $m_{h}=$ $\left(n_{\Gamma}^{2}+n_{\Gamma}\right)\left(d_{q}+3\right) / 2+\left(d_{q}+1\right)$ vectors belonging to $\mathbb{C}^{d}$ with $d=\left(n_{\Gamma}^{2}+n_{\Gamma}\right) / 2+1$. This numerical orthogonalization is carried out in using a Gram-Schmidt algorithm.

4.3 Step 2: Identification of a matrix model for the hidden variables

The last step of the hidden state variables method consists in identifying a set of matrices $\left[M_{1}^{\mathrm{opt}}\right],\left[D_{1}^{\mathrm{opt}}\right]$ and $\left[K_{1}^{\text {opt }}\right]$ such that

$$
\frac{\left[N^{\mathrm{opt}}(\omega)\right]}{q^{\mathrm{opt}}(\omega)}=\left[A_{1 \Gamma}^{\mathrm{opt}}(\omega)\right]-\left[A_{1 c}^{\mathrm{opt}}(\omega)\right]\left[A_{1 h}^{\mathrm{opt}}(\omega)\right]^{-1}\left[A_{1 c}^{\mathrm{opt}}(\omega)\right]^{T},
$$

where the symmetric $\left(n_{\Gamma} \times n_{\Gamma}\right)$ matrix $\left[A_{1 \Gamma}^{\mathrm{opt}}(\omega)\right]$, the complex $\left(n_{1}-n_{\Gamma} \times n_{\Gamma}\right)$ matrix $\left[A_{1 c}^{\mathrm{opt}}(\omega)\right]$ and the symmetric $\left(n_{1}-n_{\Gamma} \times n_{1}-n_{\Gamma}\right)$ matrix $\left[A_{1 h}^{\mathrm{opt}}(\omega)\right]$ are obtained from the block decomposition of the dynamic stiffness matrix

$$
\left[A_{1}^{\mathrm{opt}}(\omega)\right]=-\omega^{2}\left[M_{1}^{\mathrm{opt}}\right]+\jmath \omega\left[D_{1}^{\mathrm{opt}}\right]+\left[K_{1}^{\mathrm{opt}}\right],
$$


Pierre Ropars, Christophe Desceliers

which is then written as

$$
\left[A_{1}^{\mathrm{opt}}(\omega)\right]=\left(\begin{array}{cc}
{\left[A_{1 h}^{\mathrm{opt}}(\omega)\right]} & {\left[A_{1 c}^{\mathrm{opt}}(\omega)\right]} \\
{\left[A_{1 c}^{\mathrm{opt}}(\omega)\right]^{T}\left[A_{1 \Gamma}^{\mathrm{opt}}(\omega)\right]}
\end{array}\right)
$$

An algorithm presented in [15] seeks for a block diagonal matrix $\left[M_{1}^{\mathrm{opt}}\right]$ written as

$$
\left[M_{1}^{\mathrm{opt}}\right]=\left(\begin{array}{ll}
{\left[I_{n_{\mathrm{h}}}\right]} & \\
& {\left[M_{1 \Gamma}^{\mathrm{opt}}\right]}
\end{array}\right),
$$

where $\left[I_{n_{\mathrm{h}}}\right]$ is the $\left(n_{\mathrm{h}} \times n_{\mathrm{h}}\right)$ identity matrix with $n_{\mathrm{h}}=$ $d_{q} / 2$. It should be noticed that Eq. (27) is justified in [15]. Since that for any given frequency band of analysis $B_{\mathrm{f}}$, any given polynomial degree $d_{q}$ and for any given boundary dynamic stiffness matrix $\left[A_{\Gamma}\right]$, it is possible to find a set of three matrices $\left[M_{1}^{\mathrm{opt}}\right],\left[D_{1}^{\mathrm{opt}}\right]$ and $\left[K_{1}^{\mathrm{opt}}\right]$ which is constructed in solving (see $[22,19,32]$ ), then, it means that $\left(\left[M_{1}^{\mathrm{opt}}\right],\left[D_{1}^{\mathrm{opt}}\right],\left[K_{1}^{\mathrm{opt}}\right]\right)$ is the output value of a mapping $\mathcal{C}$,

$$
\left.\left(\left[M_{1}^{\mathrm{opt}}\right],\left[D_{1}^{\mathrm{opt}}\right],\left[K_{1}^{\mathrm{opt}}\right]\right)=\mathcal{C}\left(B_{\mathrm{f}} ; d_{q},\left[A_{\Gamma}\right]\right)\right) .
$$

Hence, giving the identified matrices $\left[M_{1}^{\mathrm{opt}}\right],\left[D_{1}^{\mathrm{opt}}\right]$ and $\left[K_{1}^{\mathrm{opt}}\right]$, then the matrix $\left[A_{\Gamma}^{\mathrm{opt}}(\omega)\right]$ which is defined as $\left[A_{\Gamma}^{\mathrm{opt}}(\omega)\right]=\left[A_{1 \Gamma}^{\mathrm{opt}}(\omega)\right]-\left[A_{1 c}^{\mathrm{opt}}(\omega)\right]\left[A_{1 h}^{\mathrm{opt}}(\omega)\right]^{-1}\left[A_{1 c}^{\mathrm{opt}}(\omega)\right]^{T}$ (see Eqs (25) and (26)), can be used as an approximation of the boundary dynamic boundary stiffness matrix $\left[A_{\Gamma}(\omega)\right]$.

\subsection{Remark on the numerical cost of the method}

The computational cost of the hidden state variables method is mostly due to the numerical orthogonalization of $m_{h}=\left(n_{\Gamma}^{2}+n_{\Gamma}\right)\left(d_{q}+3\right) / 2+\left(d_{q}+1\right)$ vectors belonging to $\mathbb{C}^{d}$ with $d=\left(n_{\Gamma}^{2}+n_{\Gamma}\right) / 2+1$ (see section 4.2). Consequently, the greater $n_{\Gamma}$ is and the more expensive is the hidden state variables method. For example, the Table 1 shows the computational time for performing the step 1 with respect to the number of degree of freedom $n_{\Gamma}$ on the interface $\Gamma$ for a very small problem (only 60 points in the frequency band and with $d_{q}=2$ ). Such a computational cost for performing step 1 increases exponentially and consequently such a method is extremely expensive for dynamical systems with a high number of freedom on the interface $\Gamma$.

In this paper, the proposed approach is to decrease such numeral cost in using the ad hoc functional basis presented in Section 3.
Table 1 Computational cost of step 1 against the number of degree of freedom $n_{\Gamma}$ on interface $\Gamma$.

\begin{tabular}{|c|c|}
\hline$n_{\Gamma}$ & Computational times $(\mathrm{s})$ \\
\hline 2 & 6,5538 \\
4 & 12,4342 \\
6 & 55,3256 \\
8 & 183,9891 \\
10 & 510,1938 \\
14 & 3214,5 \\
18 & 14132,0 \\
22 & 50331,0 \\
28 & 360820,0 \\
\hline
\end{tabular}

4.5 Hidden state variables method applied on the boundary dynamic stiffness matrices of $\Omega_{1}$ for each frequency band $B_{k}$

For each frequency band $B_{k}$, the hidden state variables method is applied, with a polynomial degree $d_{q, B_{k}}$, yielding the construction of matrices $\left[M_{1, B_{k}}^{\mathrm{opt}}\right],\left[D_{1, B_{k}}^{\mathrm{opt}}\right]$ and $\left[K_{1, B_{k}}^{\mathrm{opt}}\right]$ defined as

$$
\left.\left(\left[M_{1, B_{k}}^{\mathrm{opt}}\right],\left[D_{1, B_{k}}^{\mathrm{opt}}\right],\left[K_{1, B_{k}}^{\mathrm{opt}}\right]\right)=\mathcal{C}\left(B_{k} ; d_{q, B_{k}},\left[A_{\Gamma, B_{k}}\right]\right)\right) .
$$

For each frequency band $B_{k}$, a stiffness matrix $\left[A_{1, B_{k}}^{\text {opt }}(\omega)\right]$ is introduced as follows

$$
\begin{aligned}
{\left[A_{1, B_{k}}^{\mathrm{opt}}(\omega)\right] } & = \\
& -\omega^{2}\left[M_{1, B_{k}}^{\mathrm{opt}}\right]+\jmath \omega\left[D_{1, B_{k}}^{\mathrm{opt}}\right]+\left[K_{1, B_{k}}^{\mathrm{opt}}\right]
\end{aligned}
$$

The boundary stiffness matrix $\left[A_{\Gamma, B_{k}}^{\mathrm{opt}}(\omega)\right]$ is then defined as

$$
\begin{aligned}
& {\left[A_{\Gamma, B_{k}}^{\mathrm{opt}}(\omega)\right]=} \\
& \quad\left[A_{1 \Gamma, B_{k}}^{\mathrm{opt}}(\omega)\right]-\left[A_{1 c, B_{k}}^{\mathrm{opt}}(\omega)\right]\left[A_{1 h, B_{k}}^{\mathrm{opt}}(\omega)\right]^{-1}\left[A_{1 c, B_{k}}^{\mathrm{opt}}(\omega)\right]^{T},
\end{aligned}
$$

where matrices $\left[A_{1 \Gamma, B_{k}}^{\mathrm{opt}}(\omega)\right],\left[A_{1 c, B_{k}}^{\mathrm{opt}}(\omega)\right],\left[A_{1 h, B_{k}}^{\mathrm{opt}}(\omega)\right]$ and $\left[A_{1 c, B_{k}}^{\mathrm{opt}}(\omega)\right]$ are extracted from the block decomposition of matrix $\left[A_{1, B_{k}}^{\mathrm{opt}}(\omega)\right]$. We then have

$\left[A_{1, B_{k}}^{\mathrm{opt}}(\omega)\right]=\left(\begin{array}{l}{\left[A_{1 h, B_{k}}^{\mathrm{opt}}(\omega)\right]\left[A_{1 c, B_{k}}^{\mathrm{opt}}(\omega)\right]} \\ {\left[A_{1 c, B_{k}}^{\mathrm{opt}}(\omega)\right]^{T}\left[A_{1 \Gamma, B_{k}}^{\mathrm{opt}}(\omega)\right]}\end{array}\right)$

Hence, for each frequency band $B_{k}$, the reduced representation $\mathbb{u}_{2, B_{k}}(\omega)$ of $\mathbf{u}_{2, B_{k}}(\omega)$ in Eq. (15) is constructed by solving Eq. (17) and in substituting the boundary dynamic boundary stiffness matrix $\left[A_{\Gamma, B_{k}}(\omega)\right]$ in Eq.(19) by matrix $\left[A_{\Gamma, B_{k}}^{\mathrm{opt}}(\omega)\right]$. 


\section{Examples of mass, dumping and stiffness matrices identification}

In this section we propose four examples. In the two first, the mass, damping and stiffness matrices of domain $\Omega_{1}$ are identified in using the hidden state variables method that is briefly recalled in Section 4 (initial method), and in using the hidden state variables method applied on the boundary dynamic stiffness matrices of $\Omega_{1}$ for each frequency band $B_{k}$ as it is presented in Section 4.5 (modified method). In third and fourth example, only results of modified method are presented because of the lack of accuracy and because of the computational cost of the initial method.

\subsection{Description of the examples}

For the following examples, domain $\Omega$ (see Section 2) is composed of two sub-domains $\Omega_{1}$ and $\Omega_{2}$ (see Fig. 1) that are assumed to be occupied by two homogeneous isotropic viscoelastic media in $2 \mathrm{D}$ plane strains. Let $E_{1}=26 \times 10^{6} \mathrm{~Pa}, \nu_{1}=0.3, \rho_{1}=1600 \mathrm{~kg} / \mathrm{m}^{3}$ be the Young modulus, the Poisson coefficient and the mass density in $\Omega_{1}$ and let $E_{2}=26 \times 10^{9} \mathrm{~Pa}, \nu_{2}=0.257$ and $\rho_{2}=2400 \mathrm{~kg} / \mathrm{m}^{3}$ be the Young modulus, the Poisson coefficient and the mass density in $\Omega_{2}$. Two points of $\Omega_{2}$ (see Fig. 1) are blocked and the density of frequencies is 20 points per Hertz.

\subsection{Boundary frequency response function}

In order to compare the initial method and the modified method that we propose, the boundary dynamic stiffness matrix has to be written in the same functional basis. Nevertheless, it is not possible to rewrite matrices $\left[A_{\Gamma, B_{k}}^{\mathrm{opt}}(\omega)\right]$ on the same basis as $\left[A_{\Gamma}^{\mathrm{opt}}(\omega)\right]$ because we used a projection on interface displacement. It is the reason why we construct the boundary dynamic response function for each method.

As explained in Section 2, it is assumed that the numbering of the degrees of freedom is such that $\mathbf{u}_{2}(\omega)$ is written as

$$
\mathbf{u}_{2}(\omega)=\left(\begin{array}{c}
\mathbf{u}_{\Omega_{2}}(\omega) \\
\mathbf{u}_{\Gamma}(\omega)
\end{array}\right)
$$

where $\mathbf{u}_{\Omega_{2}}(\omega)$ is the vector of the $n_{2}-n_{\Gamma}$ degrees of freedom related to nodes of the finite-element mesh in $\Omega_{2}$ and $\mathbf{u}_{\Gamma}(\omega)$ is the vector of the $n_{\Gamma}$ degrees of freedom related to the nodes on interface $\Gamma$. Consequently, the block decomposition of vector $\mathbf{f}(\omega)$ of the external forces is written as

$\mathbf{f}(\omega)=\left(\begin{array}{c}\mathbf{f}_{\Omega_{2}}(\omega) \\ \mathbf{f}_{\Gamma}(\omega)\end{array}\right)$.
Let $\mathbf{f}^{j}(\omega)$ be the value of vector $\mathbf{f}(\omega)$ for which $\mathbf{f}_{\Omega_{2}}(\omega)=$ 0 and $\left\{\mathbf{f}_{\Gamma}(\omega)\right\}_{i}=\delta_{i j}$. The boundary frequency response function $\left[T_{\Gamma}(\omega)\right]$ is the complex $\left(n_{\Gamma} \times n_{\Gamma}\right)$ matrix for which the components $\left[T_{\Gamma}(\omega)\right]_{i j}$ are such that, for each $0<j \leq n_{\Gamma}$

$\left[T_{\Gamma}(\omega)\right]_{i j}=\left\{\mathbf{u}_{\Gamma}^{j}(\omega)\right\}_{i}$,

in which $\mathbf{u}_{\Gamma}^{j}(\omega)$ is the value of vector $\mathbf{u}_{\Gamma}(\omega)$ that is calculated in using Eq. (2) for which $\mathbf{u}_{2}(\omega)$ is the solution of Eq. (3) with $\mathbf{f}(\omega)=\mathbf{f}^{j}(\omega)$. The boundary frequency response function $\left[T_{\Gamma}^{\mathrm{opt}}(\omega)\right]$ is the complex $\left(n_{\Gamma} \times n_{\Gamma}\right)$ matrix for which the components $\left[T_{\Gamma}^{\mathrm{opt}}(\omega)\right]_{i j}$ are such that, for each $0<j \leq n_{\Gamma}$

$\left[T_{\Gamma}^{\mathrm{opt}}(\omega)\right]_{i j}=\left\{\mathbf{u}_{\Gamma}^{j}(\omega)\right\}_{i}$,

in which $\mathbf{u}_{\Gamma}^{j}(\omega)$ is the value of vector $\mathbf{u}_{\Gamma}(\omega)$ that is calculated in using Eq. (2) for which $\mathbf{u}_{2}(\omega)$ is the solution of Eq. (3) with $\mathbf{f}(\omega)=\mathbf{f}^{j}(\omega)$ and $\left[A_{\Gamma}(\omega)\right]=\left[A_{\Gamma}^{\mathrm{opt}}(\omega)\right]$ (see Section 4) in Eq. (4). The boundary frequency response function $\left[T_{\Gamma, B_{k}}^{\mathrm{opt}}(\omega)\right]$ is the complex $\left(n_{\Gamma} \times n_{\Gamma}\right)$ matrix for which the components $\left[T_{\Gamma, B_{k}}^{\mathrm{opt}}(\omega)\right]_{i j}$ are such that, for each $0<j \leq n_{\Gamma}$

$\left[T_{\Gamma, B_{k}}^{\mathrm{opt}}(\omega)\right]_{i j}=\left\{\mathbf{u}_{\Gamma, B_{k}}^{j}(\omega)\right\}_{i}$,

in which $\mathbf{u}_{\Gamma, B_{k}}^{j}(\omega)$ is the value of $\mathbf{u}_{\Gamma, B_{k}}(\omega)$ in Eq. (14) for which $\mathbf{q}_{B_{k}}(\omega)$ is calculated in using the second expression in Eq. (16) where $\mathbb{U}_{2, B_{k}}(\omega)$ is the solution of Eq. (17) with $\mathbb{E}_{B_{k}}(\omega)=\left[P_{B_{k}}\right]^{T} \mathbf{f}^{j}(\omega)$ and $\left[A_{\Gamma, B_{k}}(\omega)\right]=$ $\left[A_{\Gamma, B_{k}}^{\mathrm{opt}}(\omega)\right]$ (see Section 4.5) in Eq. (19). Furthermore, distances between the three boundary frequency response functions $\left[T_{\Gamma}\right],\left[T_{\Gamma}^{\mathrm{opt}}\right]$ and $\left[T_{\Gamma, B_{k}}^{\mathrm{opt}}\right]$ defined by Eq. (35) to Eq. (37) are denoted by $\varepsilon_{r}\left(\left[T_{\Gamma, B_{k}}^{\mathrm{opt}}\right],\left[T_{\Gamma}\right], B_{k}\right)$ and $\varepsilon_{r}\left(\left[T_{\Gamma}^{\mathrm{opt}}\right],\left[T_{\Gamma}\right], B_{k}\right)$ where

$\varepsilon_{r}\left(\left[T_{1}\right],\left[T_{2} \mid, B\right)=\frac{\sum_{\omega \in B}\left\|\left[T_{1}(\omega)\right]-\left[T_{2}(\omega)\right]\right\|_{F}}{\sum_{\omega \in B}\left\|\left[T_{2}(\omega)\right]\right\|_{F}}\right.$,

in which $\|[A]\|_{F}=\sqrt{\operatorname{Tr}\left([A][A]^{T}\right)}$ is the Frobenius norm of matrix $[A]$. In addition, for given degrees of freedom numbered as $i$ and $j$, we compute the following relative errors $\varepsilon_{r}\left(\left[T_{\Gamma, B_{k}}^{\mathrm{opt}}\right]_{i j},\left[T_{\Gamma}\right]_{i j}, B_{k}\right)$ and $\varepsilon_{r}\left(\left[T_{\Gamma}^{\mathrm{opt}}\right]_{i j},\left[T_{\Gamma}\right]_{i j}, B_{k}\right)$. These distances are related to a given frequency band $B_{k}$. A more global distance between $\left[T_{\Gamma}\right],\left[T_{\Gamma}^{\text {opt }}\right]$ and $\left[T_{\Gamma, B_{k}}^{\mathrm{opt}}\right]$ can be defined as

$$
\begin{aligned}
\varepsilon_{\text {modif }} & =\max _{1 \leq k \leq n_{\mathrm{f}}} \varepsilon_{r}\left(\left[T_{\Gamma, B_{k}}^{\mathrm{opt}}\right],\left[T_{\Gamma}\right], B_{k}\right), \\
\varepsilon_{\text {modif }}^{i j} & =\max _{1 \leq k \leq n_{\mathrm{f}}} \varepsilon_{r}\left(\left[T_{\Gamma, B_{k}}^{\mathrm{opt}}\right]_{i j},\left[T_{\Gamma}\right]_{i j}, B_{k}\right), \\
\varepsilon_{\text {initial }} & =\varepsilon_{r}\left(\left[T_{\Gamma}^{\mathrm{opt}}\right],\left[T_{\Gamma}\right], B_{\mathrm{f}}\right), \\
\varepsilon_{\text {initial }}^{i j} & =\varepsilon_{r}\left(\left[T_{\Gamma}^{\mathrm{opt}}\right]_{i j},\left[T_{\Gamma}\right]_{i j}, B_{\mathrm{f}}\right) .
\end{aligned}
$$




\subsection{First application, a simple case}

For this example, sub-domains $\Omega_{1}$ and $\Omega_{2}$, which model respectively a soft soil and a stiffen structure, are rectangles with dimensions $3 \times 9 \mathrm{~m}^{2}$ (subdomain $\Omega_{1}$ ) and $3 \times 3 \mathrm{~m}^{2}$ (sub-domain $\Omega_{2}$ ). The finite-element mesh of domain $\Omega_{1}$ is constituted of $6 \times 18$ quadrangles and the finite-element mesh of $\Omega_{2}$ is constituted of $6 \times 6$ quadrangles. Consequently, the number of degrees of freedom in $\Omega_{1}$ is $n_{1}=452$ and the number of degree of freedom in $\Omega_{2}$ is $n_{2}=184$. The total number of freedom in interface $\Gamma$ is $n_{\Gamma}=14$. The frequency band is $B_{\mathrm{f}}=2 \pi \times[20 ; 34] \mathrm{rad} / \mathrm{s}$.

Table 2 shows the values of $\varepsilon_{\text {modif }}, \varepsilon_{\text {modif }}^{i j}, \varepsilon_{\text {initial }}$ and $\varepsilon_{\text {initial }}^{i j}$ for $i$ and $j$ equal to $I$ or $J$ and where the degrees of freedom $\left\{\mathbf{u}_{2}\right\}_{I}$ and $\left\{\mathbf{u}_{2}\right\}_{J}$ are respectively the displacement along the horizontal axis of a point located at the center of interface $\Gamma$ and the displacement along the vertical axis of a point located at distance 0.75 meters from the center. For each computation, the number of modes per band is constant $m_{k}=m$ for all $B_{k}$, where $m$ is a chosen number of modes. The number of hidden variables, which is the half of degree $d_{q, B_{k}}=d_{q}$, is also constant for all $B_{k}$. In addition, the computational duration $t$ is given in Table 2 .

A convergence analysis with respect to $d_{q}$ has been carried out but it is not presented in this paper. Consequently, the value of $d_{q}$ at convergence depends on $m$, but in this example, $d_{q}$ is constant. In addition, results presented in Table 2 show that the values of $\varepsilon_{\text {modif }}$ and $\varepsilon_{\text {modif }}^{i j}$ tend to decrease when $m$ increases and the computational time $t$ increases with $m$. Consequently, it is possible to adjust the value of $m$ to get a compromise between values of $\varepsilon_{\text {modif }}$ or $\varepsilon_{\text {modif }}^{i j}$, and the computational cost $t$. Such adjustment is not possible for the value of $\varepsilon_{\text {initial }}$ and $\varepsilon_{\text {initial }}^{i j}$ for the initial method. A reasonable value of $m$ can be taken when $\varepsilon_{\text {modif }}$ is less than 0.01 . For this example, the values of $\varepsilon_{\text {modif }}$ and $\varepsilon_{\text {modif }}^{i j}$ with $m=12$ for the modified method are of same order than the values of $\varepsilon_{\text {initial }}$ and $\varepsilon_{\text {initial }}^{i j}$ for the initial method but the computational cost is 20 times smaller. It can be noted than the modified method yields also small values of $\varepsilon_{\text {modif }}$ for $m=4$ and for which the computational cost $t=67$ seconds which is 2900 times smaller than the computational time obtained with the initial method.

Figure 2 shows the graphs of $\left\|\left[T_{\Gamma}(\omega)\right]\right\|_{F},\left\|\left[T_{\Gamma}^{\mathrm{opt}}(\omega)\right]\right\|_{F}$ and $\left\|\left[T_{\Gamma, B_{k}}^{\mathrm{opt}}(\omega)\right]\right\|_{F}$ with $m=4$ (bottom figure), the graphs of $\left[T_{\Gamma}(\omega)\right]_{I I},\left[T_{\Gamma}^{\mathrm{opt}}(\omega)\right]_{I I}$ and $\left[T_{\Gamma, B_{k}}^{\mathrm{opt}}(\omega)\right]_{I I}$ with $m=4$ (middle figure), and finally the graphs of $\left[T_{\Gamma}(\omega)\right]_{I J}$, $\left[T_{\Gamma}^{\mathrm{opt}}(\omega)\right]_{I J}$ and $\left[T_{\Gamma, B_{k}}^{\mathrm{opt}}(\omega)\right]_{I J}$ with $m=4$ (top figure).
It can be seen that the graphs match each other and consequently the identifications of the mass, dumping and stiffness matrices by the initial method and by the modified method are very good. In addition, it shows that a mean square estimation of the distance between $\left[T_{\Gamma}(\omega)\right]_{i j},\left[T_{\Gamma}^{\mathrm{opt}}(\omega)\right]_{i j}$ and $\left[T_{\Gamma, B_{k}}^{\mathrm{opt}}(\omega)\right]_{i j}$ with $i$ and $j$ equal to $I$ or $J$ would be very small.

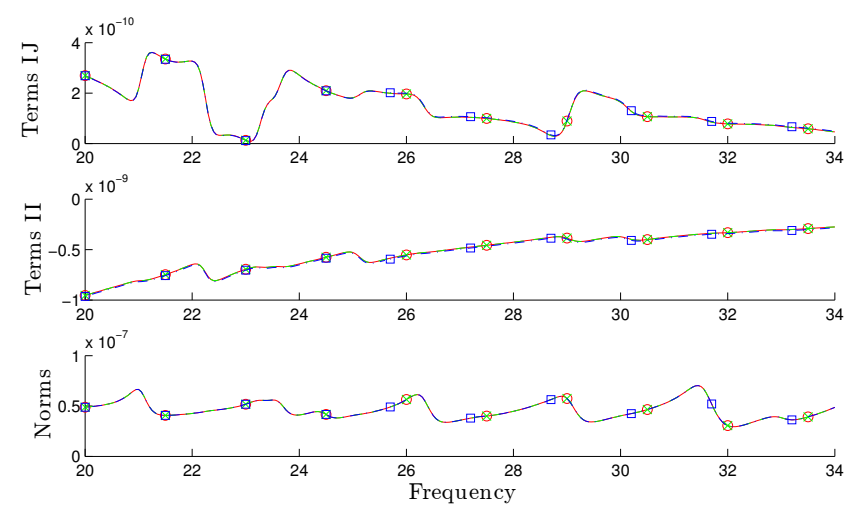

Fig. 2 Comparison between the solution of reference (finite elements method in red solid line with circular markers), the initial method (green dashed line with cross markers) and the modified method (blue dotted line with square markers) with $m=4$. Horizontal axis: $\omega$ in Hertz. Vertical axis, on the bottom graph: $\left\|\left[T_{\Gamma}(\omega)\right]\right\|_{F},\left\|\left[T_{\Gamma}^{\mathrm{opt}}(\omega)\right]\right\|_{F}$ and $\left\|\left[T_{\Gamma, B_{k}}^{\mathrm{opt}}(\omega)\right]\right\|_{F}$, on the middle graph: real parts of $\left[T_{\Gamma}(\omega)\right]_{I I},\left[T_{\Gamma}^{\mathrm{opt}}(\omega)\right]_{I I}$ and $\left[T_{\Gamma, B_{k}}^{\mathrm{opt}}(\omega)\right]_{I I}$ and on the top graph: real parts of $\left[T_{\Gamma}(\omega)\right]_{I J},\left[T_{\Gamma}^{\mathrm{opt}}(\omega)\right]_{I J}$ and $\left[T_{\Gamma, B_{k}}^{\mathrm{opt}}(\omega)\right]_{I J}$.

Moreover, for this first example, the use of several frequency bands $B_{k}\left(n_{\mathrm{f}}>1\right)$ does not bring any advantage with respect to the initial method. Nevertheless, the reduction of the displacement at the interface (Section 3.3) allows to decrease the computational cost by a factor 20 .

5.4 Second application, case with an extended frequency band $B_{\mathrm{f}}$

In the second example, sub-domains $\Omega_{1}$ and $\Omega_{2}$, which model respectively a soft soil and a stiffen structure, are rectangles with dimensions $3 \times 9 \mathrm{~m}^{2}$ (sub-domain $\Omega_{1}$ ) and $3 \times 3 \mathrm{~m}^{2}$ (sub-domain $\Omega_{2}$ ). The finite-element mesh of domain $\Omega_{1}$ is constituted of $6 \times 18$ quadrangles and the finite-element mesh of $\Omega_{2}$ is constituted of $6 \times 6$ quadrangles. Consequently, the number of degrees of freedom in $\Omega_{1}$ is $n_{1}=452$ and the number of degree of freedom in $\Omega_{2}$ is $n_{2}=184$. The total number of freedom in interface $\Gamma$ is $n_{\Gamma}=14$. The frequency band is $B_{\mathrm{f}}=2 \pi \times[20 ; 100] \mathrm{rad} / \mathrm{s}$. 
Table 3 shows the values of $\varepsilon_{\text {modif }}, \varepsilon_{\text {modif }}^{i j}, \varepsilon_{\text {initial }}$ and $\varepsilon_{\text {initial }}^{i j}$ for $i$ and $j$ equal to $I$ or $J$ and where the degrees of freedom $\left\{\mathbf{u}_{2}\right\}_{I}$ and $\left\{\mathbf{u}_{2}\right\}_{J}$ are respectively the displacement along the horizontal axis of a point located at the center of interface $\Gamma$ and the displacement along the vertical axis of a point located at distance 0.75 meters from the center. For each computations, the number of modes per band is constant $m_{k}=m$ for all $B_{k}$. The number of hidden variables, which is the half of degree $d_{q, B_{k}}=d_{q}$, is also constant for all $B_{k}$. In addition, the computational duration $t$ is given in Table 3 .

As in the first example, a convergence analysis with respect to $d_{q}$ has been carried out but it is not presented in this paper. Consequently, the value of $d_{q}$ at convergence depends on $m$, but in this example, $d_{q}$ is constant. In addition, results presented in Table 3 show that the values of $\varepsilon_{\text {modif }}$ and $\varepsilon_{\text {modif }}^{i j}$ tend to decrease when $m$ increases and the computational time $t$ increases with $m$. For this example, the values of $\varepsilon_{\text {modif }}$ and $\varepsilon_{\text {modif }}^{i j}$ with $m=12$ for the modified method are of same order than the values of $\varepsilon_{\text {initial }}$ and $\varepsilon_{\text {initial }}^{i j}$ for the initial method but the computational cost is 10 times smaller. It can be noted than the modified method yields also small values of $\varepsilon_{\text {modif }}$ for $m=6$ and for which the computational cost $t=3688$ seconds which is 345 times smaller than the computational time obtained with the initial method.

Figure 3 shows the graphs of $\left\|\left[T_{\Gamma}(\omega)\right]\right\|_{F},\left\|\left[T_{\Gamma}^{\mathrm{opt}}(\omega)\right]\right\|_{F}$ and $\left\|\left[T_{\Gamma, B_{k}}^{\mathrm{opt}}(\omega)\right]\right\|_{F}$ with $m=6$ (bottom figure), the graphs of $\left[T_{\Gamma}(\omega)\right]_{I I},\left[T_{\Gamma}^{\mathrm{opt}}(\omega)\right]_{I I}$ and $\left[T_{\Gamma, B_{k}}^{\mathrm{opt}}(\omega)\right]_{I I}$ with $m=6$ (middle figure) and finally the graphs of $\left[T_{\Gamma}(\omega)\right]_{I J}$, $\left[T_{\Gamma}^{\mathrm{opt}}(\omega)\right]_{I J}$ and $\left[T_{\Gamma, B_{k}}^{\mathrm{opt}}(\omega)\right]_{I J}$ with $m=6$ (top figure). It can be seen that the graphs match each other and consequently the identifications of the mass, dumping and stiffness matrices by the initial method and by the modified method are very good. In addition, it shows that a mean square estimation of the distance between $\left[T_{\Gamma}(\omega)\right]_{i j},\left[T_{\Gamma}^{\mathrm{opt}}(\omega)\right]_{i j}$ and $\left[T_{\Gamma, B_{k}}^{\mathrm{opt}}(\omega)\right]_{i j}$ with $i$ and $j$ equal to $I$ or $J$ would be very small. It can be noted that continuity between frequency bands $B_{k}$ is apparently respected.

In this case, the decomposition of frequency band $B_{\mathrm{f}}$ and the interface reduction allow a reduction of the computational cost by a factor 10 . In addition, for an equivalent accuracy the initial method doesn't yields causal model of boundary dynamic stiffness matrices. And consequently, the identified mass, damping and stiffness matrices can not be used for constructing a probabilistic model of random boundary dynamic stiffness matrices.

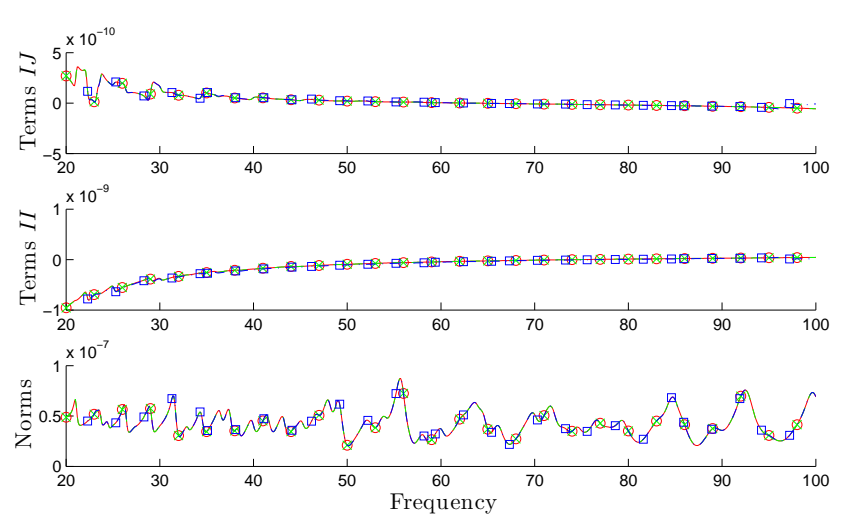

Fig. 3 Comparison between the solution of reference (finite elements method in red solid line with circular markers), the initial method (green dashed line with cross markers) and the modified method (blue dotted line with square markers) with $m=6$. Horizontal axis: $\omega$ in Hertz. Vertical axis, on the bottom graph: $\left\|\left[T_{\Gamma}(\omega)\right]\right\|_{F},\left\|\left[T_{\Gamma}^{\mathrm{opt}}(\omega)\right]\right\|_{F}$ and $\left\|\left[T_{\Gamma, B_{k}}^{\mathrm{opt}}(\omega)\right]\right\|_{F}$, on the middle graph: real parts of $\left[T_{\Gamma}(\omega)\right]_{I I},\left[T_{\Gamma}^{\mathrm{opt}}(\omega)\right]_{I I}$ and $\left[T_{\Gamma, B_{k}}^{\mathrm{opt}}(\omega)\right]_{I I}$ and on the top graph: real parts of $\left[T_{\Gamma}(\omega)\right]_{I J},\left[T_{\Gamma}^{\text {opt }}(\omega)\right]_{I J}$ and $\left[T_{\Gamma, B_{k}}^{\mathrm{opt}}(\omega)\right]_{I J}$.

5.5 Third application, case with an extended interface $\Gamma$

In this third example, sub-domains $\Omega_{1}$ and $\Omega_{2}$, which model respectively a soft soil and a stiffen structure, are rectangles with dimensions $6 \times 18 \mathrm{~m}^{2}$ (sub-domain $\Omega_{1}$ ) and $6 \times 6 \mathrm{~m}^{2}$ (sub-domain $\Omega_{2}$ ). The finite-element mesh of domain $\Omega_{1}$ is constituted of $12 \times 36$ quadrangles and the finite-element mesh of $\Omega_{2}$ is constituted of $12 \times 12$ quadrangles. Consequently, the number of degrees of freedom in $\Omega_{1}$ is $n_{1}=902$ and the number of degree of freedom in $\Omega_{2}$ is $n_{2}=366$. The total number of freedom in interface $\Gamma$ is $n_{\Gamma}=26$. The frequency band is $B_{\mathrm{f}}=2 \pi \times[20 ; 34] \mathrm{rad} / \mathrm{s}$.

Table 4 shows the values of $\varepsilon_{\text {modif }}$ and $\varepsilon_{\text {modif }}^{i j}$ for $i$ and $j$ equal to $I$ or $J$ and where the degrees of free$\operatorname{dom}\left\{\mathbf{u}_{2}\right\}_{I}$ and $\left\{\mathbf{u}_{2}\right\}_{J}$ are respectively the displacement along the horizontal axis of a point located at the center of interface $\Gamma$ and the displacement along the vertical axis of a point located at distance 1.5 meters from the center. For each computations, the number of modes per band is constant $m_{k}=m$ for all $B_{k}$, with $m$ a chosen number of modes. The number of hidden variables, which is the half of degree $d_{q, B_{k}}=d_{q}$, is also constant for all $B_{k}$. In addition, the computational duration $t$ is given in Table 4.

Results presented in Table 4 show that the values of $\varepsilon_{\text {modif }}$ and $\varepsilon_{\text {modif }}^{i j}$ tend to decrease when $m$ increases and the computational time $t$ increases with $m$. Nevertheless, for $m=1$ and $m=2$, the modal density 
leads to very small frequency bands $B_{k}$. The number of available frequency points inside such bands $B_{k}$ is then less that needed for applying the hidden state variables method. This explains numbers of band $n_{f}$ which are lower or equal than the $n_{f}$ indicated for $m=3$. It can be noted than the modified method yields also small values of $\varepsilon_{\text {modif }}$ for $m=4$ and for which the computational cost is $t=736$ seconds.

Figure 4 shows graphs of $\left\|\left[T_{\Gamma}(\omega)\right]\right\|_{F}$ and $\left\|\left[T_{\Gamma, B_{k}}^{\mathrm{opt}}(\omega)\right]\right\|_{F}$ with $m=8$ (bottom figure), the graphs of $\left[T_{\Gamma}(\omega)\right]_{I I}$ and $\left[T_{\Gamma, B_{k}}^{\mathrm{opt}}(\omega)\right]_{I I}$ with $m=8$ (middle figure) and the graphs of $\left[T_{\Gamma}(\omega)\right]_{I J}$ and $\left[T_{\Gamma, B_{k}}^{\mathrm{opt}}(\omega)\right]_{I J}$ with $m=8$ (top figure). It can be seen that the graphs match each other and consequently the identification of the mass, dumping and stiffness matrices by the modified method is good. In addition, it shows that a mean square estimation of the distance between $\left[T_{\Gamma}(\omega)\right]_{i j}$ and $\left[T_{\Gamma, B_{k}}^{\mathrm{opt}}(\omega)\right]_{i j}$ with $i$ and $j$ equal to $I$ or $J$ would be small. The identified values in the middle of Figure 4 are moved from the references values but have similar behaviour than the solution of reference.

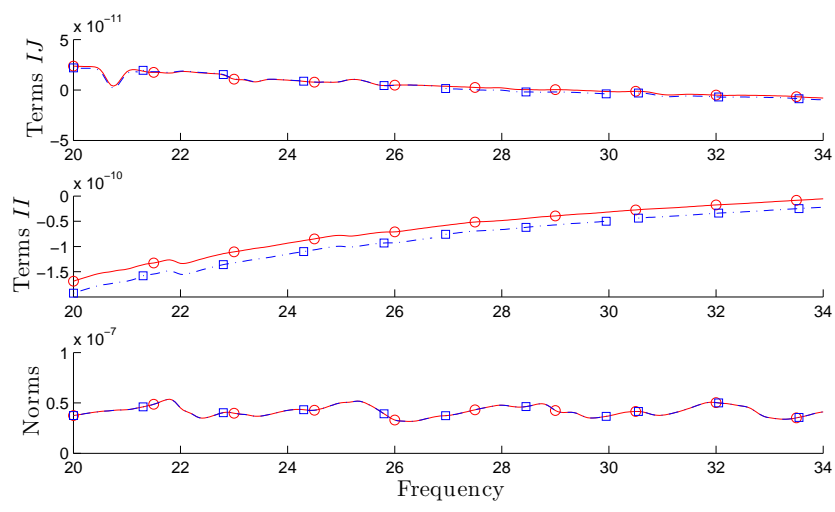

Fig. 4 Comparison between the solution of reference (finite elements method in red solid line with circular markers) and the modified method (blue dotted line with square markers) with $m=8$. Horizontal axis: $\omega$ in Hertz. Vertical axis, on the bottom graph: $\left\|\left[T_{\Gamma}(\omega)\right]\right\|_{F}$ and $\left\|\left[T_{\Gamma, B_{k}}^{\text {opt }}(\omega)\right]\right\|_{F}$, on the middle graph: real parts of $\left[T_{\Gamma}(\omega)\right]_{I I}$ and $\left[T_{\Gamma, B_{k}}^{\text {opt }}(\omega)\right]_{I I}$ and on the top graph: real parts of $\left[T_{\Gamma}(\omega)\right]_{I J}$ and $\left[T_{\Gamma, B_{k}}^{\mathrm{opt}}(\omega)\right]_{I J}$.

In this case, initial method is not able to identify the mass, damping and stiffness matrices because of a computational cost that is too much important and it is not worth it. Nevertheless, the modified method is able to identify the mass, damping and stiffness matrices which yields a causal model of boundary dynamic stiffness matrices.
5.6 Fourth application, case with an extended interface $\Gamma$ and an extended frequency band $B_{\mathrm{f}}$

In this last example, sub-domains $\Omega_{1}$ and $\Omega_{2}$, which model respectively a soft soil and a stiffen structure, are rectangles with dimensions $6 \times 18 \mathrm{~m}^{2}$ (sub-domain $\Omega_{1}$ ) and $6 \times 6 \mathrm{~m}^{2}$ (sub-domain $\Omega_{2}$ ). The finite-element mesh of domain $\Omega_{1}$ is constituted of $12 \times 36$ quadrangles and the finite-element mesh of $\Omega_{2}$ is constituted of $12 \times 12$ quadrangles. Consequently, the number of degrees of freedom in $\Omega_{1}$ is $n_{1}=902$ and the number of degree of freedom in $\Omega_{2}$ is $n_{2}=366$. The total number of freedom in interface $\Gamma$ is $n_{\Gamma}=26$. The frequency band is $B_{\mathrm{f}}=2 \pi \times[10 ; 45] \mathrm{rad} / \mathrm{s}$.

Table 5 shows the values of $\varepsilon_{\text {modif }}$ and $\varepsilon_{\text {modif }}^{i j}$ for $i$ and $j$ equal to $I$ or $J$ and where the degrees of free$\operatorname{dom}\left\{\mathbf{u}_{2}\right\}_{I}$ and $\left\{\mathbf{u}_{2}\right\}_{J}$ are respectively the displacement along the horizontal axis of a point located at the center of interface $\Gamma$ and the displacement along the vertical axis of a point located at distance 1.5 meters from the center. For each computations, the number of modes per band is constant $m_{k}=m$ for all $B_{k}$. The number of hidden variables, which is the half of degree $d_{q, B_{k}}=d_{q}$, is also constant for all $B_{k}$. In addition, the computational duration $t$ is given in Table 5 .

Results presented in Table 5 show that the values of $\varepsilon_{\text {modif }}$ and $\varepsilon_{\text {modif }}^{i j}$ tend to decrease when $m$ increases and the computational time $t$ increases with $m$. Consequently, a compromise between values of $\varepsilon_{\text {modif }}$ or $\varepsilon_{\text {modif }}^{i j}$, and the computational cost $t$ is possible. As previously, for $m=1$ and $m=2$ the frequency bands $B_{k}$ can be very small. In such cases, the density of frequencies has to be high. It can be noted than the modified method yields also small values of $\varepsilon_{\text {modif }}$ for $m=6$ and for which the computational cost is $t=8325$ seconds.

Figure 5 shows graphs of $\left\|\left[T_{\Gamma}(\omega)\right]\right\|_{F}$ and $\left\|\left[T_{\Gamma, B_{k}}^{\mathrm{opt}}(\omega)\right]\right\|_{F}$ with $m=8$ (bottom figure), the graphs of $\left[T_{\Gamma}(\omega)\right]_{I I}$ and $\left[T_{\Gamma, B_{k}}^{\mathrm{opt}}(\omega)\right]_{I I}$ with $m=8$ (middle figure) and the graphs of $\left[T_{\Gamma}(\omega)\right]_{I J}$ and $\left[T_{\Gamma, B_{k}}^{\mathrm{opt}}(\omega)\right]_{I J}$ with $m=8$ (top figure). It can be seen that the graphs match each other and consequently the identification of the mass, dumping and stiffness matrices by the modified method is good. In addition, it shows that a mean square estimation of the distance between $\left[T_{\Gamma}(\omega)\right]_{i j}$ and $\left[T_{\Gamma, B_{k}}^{\mathrm{opt}}(\omega)\right]_{i j}$ with $i$ and $j$ equal to $I$ or $J$ would be small.

In this case, initial method is not able to identify the mass, damping and stiffness matrices because of a computational cost that is too much important and it is not worth it. Nevertheless, the modified method is able to identify the mass, damping and stiffness matri- 


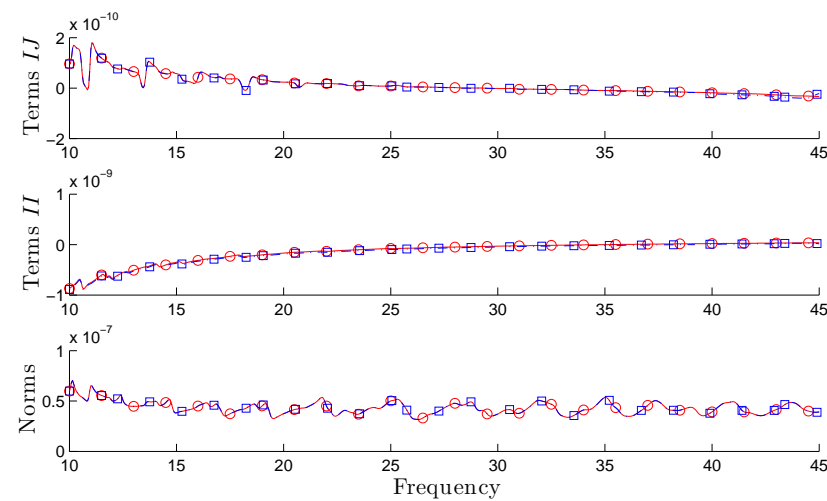

Fig. 5 Comparison between the solution of reference (finite elements method in red solid line with circular markers) and the modified method (blue dotted line with square markers) with $m=8$. Horizontal axis: $\omega$ in Hertz. Vertical axis, on the bottom graph: $\left\|\left[T_{\Gamma}(\omega)\right]\right\|_{F}$ and $\left\|\left[T_{\Gamma, B_{k}}^{\text {opt }}(\omega)\right]\right\|_{F}$, on the middle graph: real parts of $\left[T_{\Gamma}(\omega)\right]_{I I}$ and $\left[T_{\Gamma, B_{k}}^{\mathrm{opt}}(\omega)\right]_{I I}$ and on the top graph: real parts of $\left[T_{\Gamma}(\omega)\right]_{I J}$ and $\left[T_{\Gamma, B_{k}}^{\mathrm{opt}}(\omega)\right]_{I J}$.

ces which yields a causal model of boundary dynamic stiffness matrices.

\section{Probabilistic model of boundary dynamic stiffness matrix}

6.1 Random boundary dynamic stiffness matrix

For each frequency band $B_{k}$, we define the random stiffness matrix $\left[\mathbf{A}_{1, B_{k}}(\omega)\right]$ as

$$
\left[\mathbf{A}_{1, B_{k}}(\omega)\right]=-\omega^{2}\left[\mathbf{M}_{1, B_{k}}\right]+\jmath \omega\left[\mathbf{D}_{1, B_{k}}\right]+\left[\mathbf{K}_{1, B_{k}}\right],
$$

where random matrices $\left[\mathbf{M}_{1, B_{k}}\right],\left[\mathbf{D}_{1, B_{k}}\right]$ and $\left[\mathbf{K}_{1, B_{k}}\right]$ belong to the stochastic set of random matrices $\mathrm{SE}^{+}$ introduced by C. Soize in $[14,13]$. Introducing those random matrices allows the uncertainties related to the modelling of matrices $\left[M_{1, B_{k}}^{\mathrm{opt}}\right],\left[D_{1, B_{k}}^{\mathrm{opt}}\right]$ and $\left[K_{1, B_{k}}^{\mathrm{opt}}\right]$ to be taken into account. Their probabilistic models depend on three dispersion parameters $\delta_{M, B_{k}}, \delta_{D, B_{k}}, \delta_{K, B_{k}}$ which control the level of uncertainties related to $\left[M_{1, B_{k}}^{\text {opt }}\right]$, $\left[D_{1, B_{k}}^{\mathrm{opt}}\right]$, and $\left[K_{1, B_{k}}^{\mathrm{opt}}\right]$. In addition random matrices $\left[\mathbf{M}_{1, B_{k}}\right],\left[\mathbf{D}_{1, B_{k}}\right]$, and $\left[\mathbf{K}_{1, B_{k}}\right]$ are written as

$$
\begin{aligned}
& {\left[\mathbf{M}_{1, B_{k}}\right]=\left[L_{M, B_{k}}^{\mathrm{opt}}\right]\left[\mathbf{G}_{M, B_{k}}\right]\left[L_{M, B_{k}}^{\mathrm{opt}}\right]^{T},} \\
& {\left[\mathbf{D}_{1, B_{k}}\right]=\left[L_{D, B_{k}}^{\mathrm{opt}}\right]\left[\mathbf{G}_{D, B_{k}}\right]\left[L_{D, B_{k}}^{\mathrm{opt}}\right]^{T},} \\
& {\left[\mathbf{K}_{1, B_{k}}\right]=\left[L_{K, B_{k}}^{\mathrm{opt}}\right]\left[\mathbf{G}_{K, B_{k}}\right]\left[L_{K, B_{k}}^{\mathrm{opt}}\right]^{T},}
\end{aligned}
$$

in which matrices $\left[M_{1, B_{k}}\right]=\left[L_{M, B_{k}}^{\mathrm{opt}}\right]\left[L_{M, B_{k}}^{\mathrm{opt}}\right]^{T},\left[D_{1, B_{k}}\right]=$ $\left[L_{D, B_{k}}^{\mathrm{opt}}\right]\left[L_{D, B_{k}}^{\mathrm{opt}}\right]^{T}$, and $\left[K_{1, B_{k}}\right]=\left[L_{K, B_{k}}^{\mathrm{opt}}\right]\left[L_{K, B_{k}}^{\mathrm{opt}}\right]^{T}$ are the Cholesky factorizations of matrices $\left[M_{1, B_{k}}^{\mathrm{opt}}\right],\left[D_{1, B_{k}}^{\mathrm{opt}}\right]$, and $\left[K_{1, B_{k}}^{\text {opt }}\right]$ and in which the random real $\left(m_{k} \times m_{k}\right)$ matrices $\left[\mathbf{G}_{M, B_{k}}\right],\left[\mathbf{G}_{D, B_{k}}\right]$, and $\left[\mathbf{G}_{K, B_{k}}\right]$ belong to the set $\mathrm{SG}^{+}$introduced by C. Soize in $[14,13]$. A probabilistic model of uncertainties related to the boundary dynamic stiffness matrices $\left[A_{\Gamma}(\omega)\right]$ is then constructed for each frequency band $B_{k}$ in substituting the deterministic matrix $\left[A_{B_{k}}(\omega)\right]$ in Eq. (17) by a random matrix $\left[\mathbf{A}_{B_{k}}(\omega)\right]$ and in substituting the deterministic vector $\mathbb{U}_{2, B_{k}}(\omega)$ by a random vector $\mathbb{U}_{2, B_{k}}(\omega)$ such that

$\left(\left[A_{2, B_{k}}(\omega)\right]+\left[\mathbf{A}_{B_{k}}(\omega)\right]\right) \mathbb{U}_{2, B_{k}}(\omega)=\mathbb{E}_{B_{k}}(\omega)$,

The random matrix $\left[\mathbf{A}_{B_{k}}(\omega)\right]$ is written as

$\left[\mathbf{A}_{B_{k}}(\omega)\right]=\left(\begin{array}{ll}{\left[0_{2}\right]} & \\ & {\left[\mathbf{A}_{\Gamma, B_{k}}(\omega)\right]}\end{array}\right)$,

in which $\left[\mathbf{A}_{\Gamma, B_{k}}(\omega)\right]$ is a random symmetric $\left(m_{B_{k}}^{+} \times\right.$ $\left.m_{B_{k}}^{+}\right)$matrix such that

$$
\begin{aligned}
& {\left[\mathbf{A}_{\Gamma, B_{k}}(\omega)=\right.} \\
& {\left[\mathbf{A}_{1 \Gamma, B_{k}}(\omega)\right]-\left[\mathbf{A}_{1 c, B_{k}}(\omega)\right]\left[\mathbf{A}_{1 h, B_{k}}(\omega)\right]^{-1}\left[\mathbf{A}_{1 c, B_{k}}(\omega)\right]^{T},}
\end{aligned}
$$

where random complex matrices $\left[\mathbf{A}_{1 \Gamma, B_{k}}(\omega)\right],\left[\mathbf{A}_{1 c, B_{k}}(\omega)\right]$, $\left[\mathbf{A}_{1 h, B_{k}}(\omega)\right]$ and $\left[\mathbf{A}_{1 c, B_{k}}(\omega)\right]$ are extracted from the following block decomposition of random matrix $\left[\mathbf{A}_{1, B_{k}}(\omega)\right]$

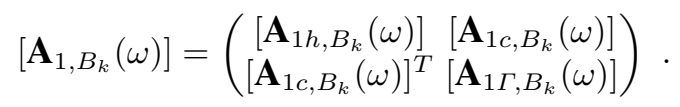

Consequently, for each frequency band $B_{k}$, the finite element vector of the degree of freedom in domain $\Omega_{2}$ is modelled by a random vector $\mathbf{U}_{2, B_{k}}(\omega)$ which is constructed in substituting $\mathbb{U}_{2, B_{k}}(\omega)$ by $\mathbb{U}_{2, B_{k}}(\omega)$ in Eq. (15). We then have

$\mathbf{U}_{2, B_{k}}(\omega)=\left[P_{B_{k}}\right] \mathbb{U}_{2, B_{k}}(\omega)$.

It should be noted that without any additional information concerning the statistics between $\left[\mathbf{A}_{\Gamma, B_{k}}(\omega)\right]$ and $\left[\mathbf{A}_{\Gamma, B_{\ell}}(\omega)\right]$ with $\ell \neq k$ then, for all $\ell$ and $k$, matrices $\left[\mathbf{G}_{M, B_{k}}\right],\left[\mathbf{G}_{M, B_{\ell}}\right],\left[\mathbf{G}_{D, B_{k}}\right],\left[\mathbf{G}_{D, B_{\ell}}\right],\left[\mathbf{G}_{K, B_{k}}\right]$, and, $\left[\mathbf{G}_{K, B_{\ell}}\right]$ are six statistical independent random matrices for all $\ell$ and $k$.

6.2 Example of random boundary frequency response function

For same case as in section 5.3, we compute 200 stochastic realizations of random boundary dynamic stiffness matrix $\left[\mathbf{A}_{\Gamma, B_{k}}(\omega)\right]$. It allows to construct 200 random boundary frequency response matrices $\left[\mathbf{T}_{\Gamma, B_{k}}(\omega)\right]$ by Eq. (37) where the displacement interpolation is obtained by Eq. (51). The number of modes per band $m$ is chosen as $m=6$ and dispersion parameters are equal, $\delta_{M, B_{k}}=\delta_{D, B_{k}}=\delta_{K, B_{k}}=0.3$ for both frequency bands 
$B_{k}\left(n_{f}=2\right)$.

Figure 6 shows three parts, relative to the norm of the boundary frequency response matrix $\left\|\left[\mathbf{T}_{\Gamma, B_{k}}^{\text {opt }}(\omega)\right]\right\|_{F}$, and the two components $\left[\mathbf{T}_{\Gamma, B_{k}}^{\text {opt }}(\omega)\right]_{I I}$ and $\left[\mathbf{T}_{\Gamma, B_{k}}^{\text {opt }}(\omega)\right]_{I J}$. On the three parts, the values of reference $\left\|\left[T_{\Gamma}(\omega)\right]\right\|_{F},\left[T_{\Gamma}(\omega\right.$ and $\left[T_{\Gamma}(\omega)\right]_{I J}$ are plotted in red lines. One stochastic realization of $\left\|\left[\mathbf{T}_{\Gamma, B_{k}}^{\mathrm{opt}}(\omega)\right]\right\|_{F},\left[\mathbf{T}_{\Gamma, B_{k}}^{\mathrm{opt}}(\omega)\right]_{I I}$ and $\left[\mathbf{T}_{\Gamma, B_{k}}^{\mathrm{opt}}(\omega)\right.$ are also presented in dashed black line. In addition, deterministic values $\left\|\left[T_{\Gamma, B_{k}}^{\mathrm{opt}}(\omega)\right]\right\|_{F},\left[T_{\Gamma, B_{k}}^{\mathrm{opt}}(\omega)\right]_{I I}$ and $\left[T_{\Gamma, B_{k}}^{\mathrm{opt}}\right.$ are plotted in dashed blue lines. The $90 \%$ confidence intervals computed with 200 realizations are shown in green solid line. With Figure 6, it can be noted that constant $\delta$ parameters on $B_{\mathrm{f}}$ seems allow the keeping of the continuity on each stochastic realizations (near $29 \mathrm{~Hz})$. Because of the large values of $\delta_{M, B_{k}}, \delta_{D, B_{k}}$ and $\delta_{K, B_{k}}$, the dispersion indicated by the $90 \%$ confidence interval is large compared with the variation of deterministic values. The dispersion in Forbenius norm is less accented, but respects the aspect of the dispersions of components.

The new stochastic model exposed in this numerical example is not similar to the initial model. The crosscorrelation between the different frequency bands have no meaning. In state, the stochastic model allows to choose different deviation parameters for each groups of eigen modes. This can be interpreted by a variation of the own dimensions of the structure. It allows to measure the impact of variations of the eigen modes of the structure on its dynamical behaviour.

\section{Conclusion}

We present a reduction method of the hidden state variables method which is subdivided on smallest frequency bands with restrained number of modes. The polynomial bases, used in the identification process, are now dimensioned by the number of modes chosen for each sub-problems. Besides the fact that each sub-problem can be treated in parallel, substantial gain of the computational time is possible. For a equivalent precision the computational cost is 10 to 20 times less than in the initial method. For an acceptable precision, the computational cost could be more than 1000 times smaller. Then, we show that the loss of precision is acceptable in terms of spared time. In addition, when size of the boundary dynamical stiffness matrix grows up, the precision stay good with enough modes. In such cases initial method is not able to identify the mass, damping and stiffness matrices because of a computational cost that is too much important.

Nevertheless, the approach needs several eigen modes and a sufficient density of frequencies in respect to the
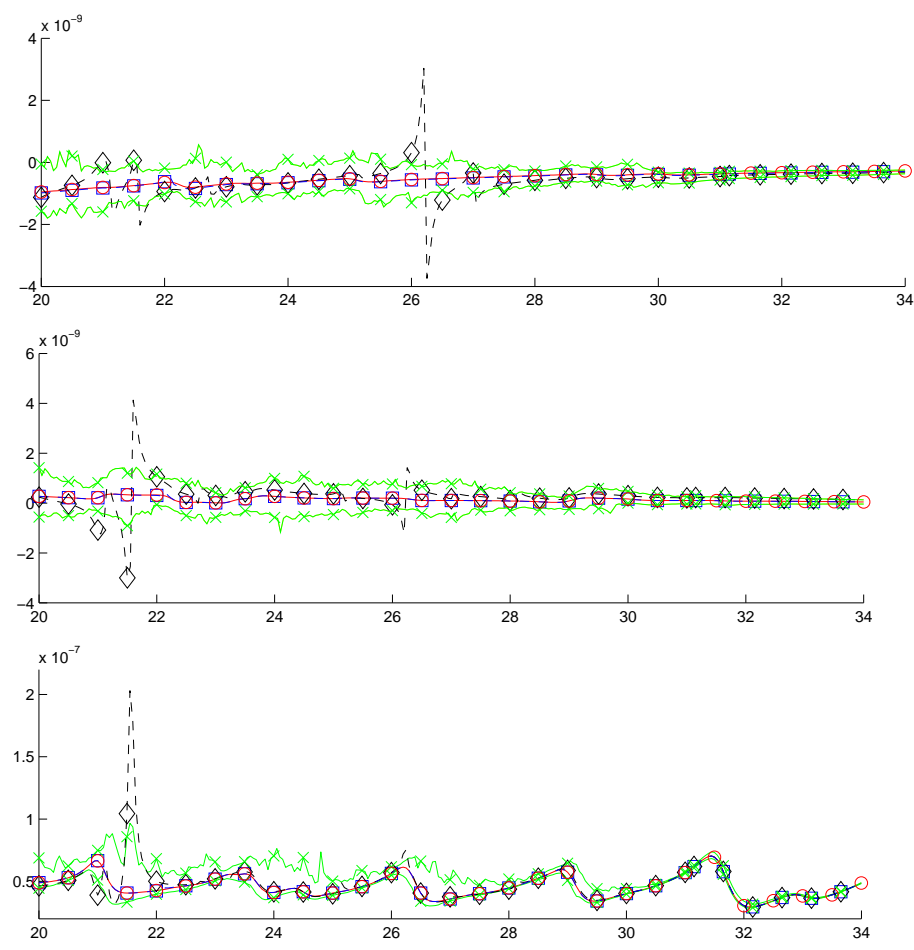

Fig. 6 Statistical results; Horizontal axis: $\omega$ in Hertz. Vertical axis, on bottom graph norms of: matrix of reference (red solid line with circular markers) $\left\|\left[T_{\Gamma}(\omega)\right]\right\|_{F}$, deterministic reduced matrix (blue dotted line with square markers) $\left\|\left[T_{\Gamma, B_{k}}^{\text {opt }}(\omega)\right]\right\|_{F}$, a random realization of $\left\|\left[\mathbf{T}_{\Gamma, B_{k}}^{\text {opt }}(\omega)\right]\right\|_{F}$ (black dashed line with diamond markers) and $90 \%$ confidence interval (green solid lines with cross markers), on middle graph the $I I$ components of: $\left[T_{\Gamma}(\omega)\right]$ (red solid line with circular markers), $\left[T_{\Gamma, B_{k}}^{\mathrm{opt}}(\omega)\right]$ (blue dotted line with square markers), $\left\|\left[\mathbf{T}_{\Gamma, B_{k}}^{\text {opt }}(\omega)\right]\right\|_{F}$ (black dashed line with diamond markers) and 90\% confidence interval (green sold line with cross markers), on top graph the $I J$ components of: $\left[T_{\Gamma}(\omega)\right]$ (red solid line with circular markers), $\left[T_{\Gamma, B_{k}}^{\mathrm{opt}}(\omega)\right]$ (blue dotted line with square markers), $\left.\left[\mathbf{T}_{\Gamma, B_{k}}^{\text {opt }}(\omega)\right]\right|_{F}$ (black dashed line with diamond markers) and 90\% confidence interval (green sold line with cross markers).

density of modes. However, further study should be done to obtain equivalent statistical characteristics as the initial method.

\section{References}

1. O. C. Zienkiewicz, R. L. Taylor and J. Z. Zhu (2005) The finite element method, volume 1. Butterworth-Heinemann, sixth edition.

2. D. Givoli (1992) Numerical Methods for Problems in Infinite Domains. Studies in Applied Mechanics. Elsevier.

3. J. P. Wolf and C. Song (1996) Finite-element modelling of unbounded media. Wiley, Chichester.

4. E. Turkel (1998) Special issue on absorbing boundary conditions. Applied Numerical Mathematics, 27(4):327-560.

5. F. Magoulès and I. Harari (2006) Absorbing boundary conditions. Computer Methods in Applied Mechanics and Engineering, 195(29-32):3551-3902. 
6. S. V. Tsynkov (1998) Numerical solution of problems on unbounded domains. a review. Applied Numerical Mathematics, 27:465-532.

7. M. Bonnet (1999) Boundary Integral Equation Methods for Solids and Fluids. Wiley-Blackwell.

8. J. P. Wolf and C. Song (2001) The scaled boundary finiteelement method - a fundamental solution-less boundaryelement method. Computer Methods in Applied Mechanics and Engineering, 190(42):5551-5568.

9. J. Mondot, B. Petersson (1987) Characterization of strutureborne sound sources: The source descriptor and the coupling function. Journal of sound and vibration 114:507-518.

10. Y. Koh, R. White (1996) Analysis and control of vibration power transmission to machinery supporting structures subjected to a multi-excitation system, Part I: Driving point mobility matrix of beams and rectangular plates. Journal of sound and vibration 196:469-493.

11. M. Villot, P. Ropars, E. Bongini, et al (2011) Modelling the influence of structural modifications on the response of a building to railway vibration. Noise control Engineering Journal 59:641-651.

12. A. Cherukuri, P. E. Barbone (1998) High modal density approximation for equipment in the time domain. Journal of Acoustical Society of America 104(2):2048-2053.

13. C. Soize (2000) A nonparametric model of random uncertainties for reduced matrix models in structural dynamics. Probabilistic engineering mechanics 15:277-294.

14. C. Soize (2005) Random matrix theory for modeling uncertainties in computational mechanics. Computer methods in applied mechanics and engineering 194:1333-1366.

15. R. Cottereau, D. Clouteau, C. Soize (2007) Construction of a probabilistic model for impedance matrices. Comput. Methods Appl. Mech. Engrg. 196:2252-2268.

16. R. Cottereau, D. Clouteau, C. Soize (2006) Probabilistic nonparametric model of impedance matrices: Application to the seismic design of a structure. European Journal of Computational Mechanics 15:131-142.

17. P. Ropars, C. Desceliers, P. Jean (2014) Quantification of uncertainties in a computational model of interaction soilstructure with a railway excitation. submit on October 2014 in Journal of Vibration and Acoustics.

18. F. Chabas, C. Soize (1987) Modeling mechanical subsystems by boundary impedance in the finite element method. La Recherche Aérospatiale (english edition), 5:59-75.

19. P. Ropars, G. Bonnet, P. Jean (2014) A stabilization process applied to a hidden variables method for evaluating the uncertainties on foundation impedances and their effect on vibrations induced by railways in a building. Journal of Sound and Vibration 333(1):1953-1971.

20. J. W. S. Rayleigh (1945) The theory of sound. Volume 1, Diver Publications, New York.

21. A. Dienstfrey, L. Greengard (2001) Analytic continuation, singular-value expansions, and Kramers-Kronig analysis. Inverse Problems 17:1307-1320.

22. R. Cottereau, D. Clouteau, C. Soize (2007) Modèle dynamique équivalent de matrices d'impédance de fondation. $7^{\text {ème }}$ Colloque National, AFPS 2007 - École Centrale Paris.

23. M. I. Friswell (1990) Candidate reduced order models for structural parameter estimation. Journal of Vibration and Acoustics 112:93-97.

24. R. R. Craig (1995) Substructure methods in vibration. Journal of Vibration and Acoustics 117(3):207-203.

25. S. Chaillat and M. Bonnet (2014) A new Fast Multipole formulation for the elastodynamic half-space Green's tensor. Journal of Computational Physics, 258:787-808.
26. M. H. Bazyar and C. Song (2008) A continued-fraction-based high-order transmitting boundary for wave propagation in unbounded domains of arbitrary geometry. International Journal for Numerical Methods in Engineering 74:209-237.

27. S. Tang, T. Y. Hou and W. K. Liu (2006) A mathematical framework of the bridging scale method. International Journal for Numerical Methods in Engineering 65:1688-1713.

28. P. E. Barbone, A. Cherukuri, D. Goldman (2000) Canonical representation of complex vibratory subsystems: time domain Dirichlet to Neumann maps. International Journal of Solids and Structures 37:2825-2857.

29. P. E. Barbone, D. Givoli, I. Patlashenko (2003) Optimal modal reduction of vibrating substructures. International Journal for Numerical Methods in Engineering 57:341-369.

30. D. Givoli, P. E. Barbone, I. Patlashenko (2004) Which are the important modes of a subsystem? International Journal for Numerical Methods in Engineering, 59:1657-1678.

31. C. Sanathanan, J. Koerner (1963) Transfer function synthesis as a ratio of two complex polynomials. IEEE Transaction on Automatic Control 56-58.

32. X. Du, M. Zhao (2009) Stability and identification for rational approximation of frequency response function of unbounded soil. Earthquake Engineering and Structural Dynamic 39:165-186. 
Table 2 Convergence analysis with respect to the number of modes per band $m$

\begin{tabular}{|c|c|c|l|l|l|l|r|}
\hline$m$ & $n_{f}$ & $d_{q}$ & $\varepsilon_{\text {modif }}$ & $\varepsilon_{\text {modif }}^{I I}$ & $\varepsilon_{\text {modif }}^{J J}$ & $\varepsilon_{\text {modif }}^{I J}$ & $t(s)$ \\
\hline 1 & 7 & 4 & 0,4667 & 0,9893 & 0,8490 & 0,9868 & 27 \\
2 & 4 & 4 & 0,1025 & 0,9957 & 0,9832 & 0,9926 & 37 \\
3 & 3 & 4 & 0,0248 & 0,4244 & 0,9767 & 2,0145 & 55 \\
4 & 2 & 4 & 0,0003206 & 0,0454 & 0,0160 & 0,0088 & 67 \\
6 & 2 & 4 & 0,0001670 & 0,0412 & 0,0051 & 0,0176 & 255 \\
8 & 1 & 4 & 0,00000895 & 0,0213 & 0,0015 & 0,0010 & 954 \\
10 & 1 & 4 & 0,00000608 & 0,0261 & 0,0009 & 0,0127 & 2759 \\
12 & 1 & 4 & 0,00000296 & 0,0028 & 0,0004 & 0,0047 & 9747 \\
\hline & & & & & & & \\
$n_{\Gamma}$ & $n_{f}$ & $d_{q}$ & $\varepsilon_{\text {intial }}$ & $\varepsilon_{\text {initial }}^{I I}$ & $\varepsilon_{\text {initial }}^{J J}$ & $\varepsilon_{\text {initial }}^{I J}$ & $t(s)$ \\
\hline 14 & 1 & 4 & 0,000000509 & 0,0000677 & 0,0003452 & 0,0008712 & 194234 \\
\hline
\end{tabular}

Table 3 Convergence study of the reduced method in respect to number of modes per band $m$

\begin{tabular}{|c|c|c|l|r|r|r|r|}
\hline$m$ & $n_{f}$ & $d_{q}$ & $\varepsilon_{\text {modif }}$ & $\varepsilon_{\text {modif }}^{I I}$ & $\varepsilon_{\text {modif }}^{J J}$ & $\varepsilon_{\text {modif }}^{I J}$ & $t(s)$ \\
\hline 1 & 42 & 4 & 0,5693 & 162,9618 & 10,3921 & 206,3245 & 154 \\
2 & 30 & 4 & 0,1813 & 11,4529 & 8,1665 & 32,5976 & 321 \\
3 & 21 & 4 & 0,0445 & 4,8319 & 10,2162 & 38,6399 & 852 \\
4 & 16 & 4 & 0,0135 & 8,7591 & 2,5721 & 30,5562 & 1016 \\
6 & 11 & 4 & 0,0055 & 1,0313 & 1,1461 & 1,4587 & 3688 \\
8 & 8 & 4 & 0,000475 & 0,6813 & 0,2395 & 0,2296 & 12367 \\
10 & 8 & 4 & 0,000218 & 0,5239 & 0,2114 & 0,2037 & 40863 \\
12 & 6 & 4 & 0,0000386 & 0,1584 & 0,0147 & 0,0244 & 130725 \\
\hline & & & & $\varepsilon_{\text {initial }}^{I I}$ & $\varepsilon_{\text {initial }}^{J J}$ & $\varepsilon_{\text {initial }}^{I J}$ & $t(s)$ \\
$n_{\Gamma}$ & $n_{f}$ & $d_{q}$ & $\varepsilon_{\text {intial }}$ & & & & \\
\hline 14 & 1 & 8 & 0,0000084588 & 0,0011 & 0,0002 & 0,0011 & 1272042 \\
\hline
\end{tabular}

Table 4 Convergence study of the reduced method in respect to number of modes per band $m$

\begin{tabular}{|c|c|c|l|r|r|r|r|}
\hline$m$ & $n_{f}$ & $d_{q}$ & $\varepsilon_{\text {modif }}$ & $\varepsilon_{\text {modif }}^{I I}$ & $\varepsilon_{\text {modif }}^{J J}$ & $\varepsilon_{\text {modif }}^{I J}$ & $t(s)$ \\
\hline 1 & 8 & 4 & 0,2638 & 13,2821 & 0,9994 & 42,4894 & 106 \\
2 & 9 & 4 & 1,2161 & 1,6721 & 11,9184 & 82,8458 & 199 \\
3 & 9 & 4 & 0,1125 & 5,2505 & 1,9853 & 11,2665 & 617 \\
4 & 7 & 4 & 0,0014 & 2,5034 & 0,8715 & 21,3065 & 736 \\
6 & 5 & 4 & 0,0004933 & 1,5117 & 0,1491 & 3,0762 & 3765 \\
8 & 4 & 4 & 0,0002532 & 1,0135 & 0,0853 & 1,2228 & 9310 \\
10 & 3 & 4 & 0,0001112 & 0,8393 & 0,0705 & 0,2563 & 27709 \\
12 & 3 & 4 & 0,0000533 & 0,7776 & 0,0368 & 0,1006 & 132549 \\
\hline
\end{tabular}

Table 5 Convergence study of the reduced method in respect to number of modes per band $m$

\begin{tabular}{|c|c|c|l|r|l|r|r|}
\hline$m$ & $n_{f}$ & $d_{q}$ & $\varepsilon_{\text {modif }}$ & $\varepsilon_{\text {modif }}^{I I}$ & $\varepsilon_{\text {modif }}^{J J}$ & $\varepsilon_{\text {modif }}^{I J}$ & $t(s)$ \\
\hline 1 & 14 & 4 & 1,6641 & 114,1307 & 5,7465 & 875,0511 & 198 \\
2 & 21 & 4 & 1,4433 & 144,4693 & 7,3431 & 49,5112 & 387 \\
3 & 22 & 4 & 0,9317 & 57,1797 & 7,0027 & 47,8213 & 1251 \\
4 & 17 & 4 & 0,2688 & 12,3305 & 3,2065 & 20,3804 & 1522 \\
6 & 12 & 4 & 0,0048 & 7,6171 & 0,9880 & 4,5339 & 8325 \\
8 & 9 & 4 & 0,0028 & 1,6416 & 0,9888 & 1,3611 & 22552 \\
10 & 8 & 4 & 0,00080232 & 1,0051 & 0,1582 & 0,2669 & 69114 \\
12 & 7 & 4 & 0,00026208 & 1,1289 & 0,1391 & 0,0952 & 415586 \\
\hline
\end{tabular}

